\title{
TREE-LEVEL INVARIANTS OF THREE-MANIFOLDS, MASSEY PRODUCTS AND THE JOHNSON HOMOMORPHISM
}

\author{
STAVROS GAROUFALIDIS AND JEROME LEVINE
}

Dedicated to Dennis Sullivan on the occasion of his 60th birthday

\begin{abstract}
We show that the tree-level part of a theory of finite type invariants of 3-manifolds (based on surgery on objects called claspers, Y-graphs or clovers) is essentially given by classical algebraic topology in terms of the Johnson homomorphism and Massey products, for arbitrary 3-manifolds. A key role of our proof is played by the notion of a homology cylinder, viewed as an enlargement of the mapping class group, and an apparently new Lie algebra of graphs colored by $H_{1}(\Sigma)$ of a closed surface $\Sigma$, closely related to deformation quantization on a surface [AMR1, AMR2, Ko3] as well as to a Lie algebra that encodes the symmetries of Massey products and the Johnson homomorphism. In addition, we give a realization theorem for Massey products and the Johnson homomorphism by homology cylinders.
\end{abstract}

\section{INTRODUCTION}

1.1. A brief summary. In this paper we investigate relations between three different phenomena in low-dimensional topology:

(a) Massey products on the first cohomology $H^{1}(M)$ with integer coefficients of 3-manifolds $M$.

(b) the Johnson homomorphism on the mapping class group of an orientable surface

(c) the Goussarov-Habiro theory of finite-type invariants of 3-manifolds.

A key point of the connection between (a) and (b) is the notion of a homology cylinder, i.e., a homology cobordism between an orientable surface and itself. This notion generalizes the mapping class group of a surface (in that case the cobordism is a product). We will construct an extension of the Johnson homomorphism to homology cylinders and use it to completely determine, in an explicit fashion, the possible Massey products at the first non-trivial level in a closed 3-manifold (assuming that the first homology $H_{1}$ is torsion-free) - see Theorem 1, Corollary 2.2 and Theorem 4 .

This generalizes the known relationship between the Johnson homomorphism and Massey products in the mapping torus of a diffeomorphism of a surface to the more general situation of homology cylinders - see Theorems 2, 3 and Remark 4.12.

For historical reasons, we should mention early work of Sullivan $[\mathrm{Su}]$ on a relation between (a) and (b), and, for an alternative point of view, work of Turaev [Tu].

With regards to the connection between (b) and (c), the main idea is to consider Massey products as finite-type invariants of 3-manifolds, and to interpret them by a graphical calculus on trees - see

\footnotetext{
Date: July 25, $2003 \quad$ First edition: March 8, 1999.

The authors were partially supported by NSF grants DMS-98-00703 and DMS-96-26639 respectively, and by an Israel-US BSF grant.

This and related preprints can also be obtained at http://www.math.gatech.edu/ stavros and http://www.math. brandeis.edu/Faculty/jlevine/

1991 Mathematics Classification. Primary 57N10. Secondary 57M25.

Key words and phrases: Massey products, lower central series, finite type invariants, homology cylinders, Johnson's homomorphism, deformation quantization, graph cohomology.
} 
Theorem 7-in much the same way that Vassiliev invariants of links have a graphical representation and that Milnor's $\mu$-invariants are known to be exactly the Vassiliev invariants of (concordance classes of) string links which are represented by trees, see $[\mathrm{HM}]$. A by-product of this investigation is a curious Lie algebra structure on a vector space of the graphs which describe finite type invariants of homology cylinders - see Proposition 2.8 and Theorem 5-that corresponds to the stacking of one homology cylinder on top of another, and is closely related to deformation quantization on a surface [AMR1, AMR2, Ko3].

1.2. History. Years ago, Johnson introduced a homomorphism (the so-called Johnson homomorphism) which he used to study the mapping class group, [Jo1, Mo4]. Morita [Mo1] discovered a close relation between the Johnson homomorphism and the simplest finite type invariant of 3-manifolds, namely the Casson invariant; this relation was subsequently generalized by the authors [GL1, GL2] to all finite type invariants of integral homology 3-spheres (i.e., 3-manifolds $M$ with $H_{1}(M, \mathbb{Z})=0$ ). This generalization posed the question of understanding the Johnson homomorphism (crucial to the structure of the mapping class group) from the point of view of finite type invariants. Unfortunately, this question is rather hard to answer if we confine ourselves to invariants of integral homology 3-spheres. This difficulty is overcome by using a theory of finite type invariants based on the notion of surgery on $Y$-links, see [Gu1, Gu2, Hb, Oh, GGP]. Using this theory we will show that the Johnson homomorphism is contained in its tree-level part, and we conjecture that an extension of the Johnson homomorphism to homology cylinders (i.e., 3-manifolds with boundary that homologically look like the product of a surface with $[0,1]$ ), which we define below, gives the full tree-level part; thus answering questions raised by Hain and Morita [Ha, Mo4].

En route to answering the above question, we were led to study this theory of invariants for homology cylinders (studied also from a slightly different perspective by Goussarov [Gu1, Gu2] and Habiro $[\mathrm{Hb}]$ ) and discovered an apparently new Lie algebra of graphs colored by $H_{1}(\Sigma)$ of a closed surface $\Sigma$, closely related to deformation quantization on a surface [AMR1, AMR2], and to the curious graded group $\mathrm{D}(A) \stackrel{\text { def }}{=} \operatorname{Ker}(A \otimes \mathrm{L}(A) \rightarrow \mathrm{L}(A)$ ), (where $\mathrm{L}(A)$ denotes the free Lie ring of a torsion-free abelian group $A$ ) studied independently by several authors with a variety of motivations [Jo2, Mo2, Ih, Dr, Ko1, Ko2, O1, O2, HM].

It turns out that Massey products of 3-manifolds naturally take values in $\mathrm{D}(A)$, and so does the Johnson homomorphism, which is also closely related to Massey products - a fact well-known to Johnson [Jo2], and later proved by Kitano [Ki]. However it is now known that the Johnson homomorphism cannot realize all elements of $\mathrm{D}(A)$, but we will see that one can achieve this realizability by replacing surface diffeomorphisms by homology cylinders.

The generalized Johnson homomorphism actually provides universally-defined invariants of homology cylinders, lifting the only partially-defined Massey products. These are our explicit candidates for the full tree-level part of the Goussarov-Habiro theory (for homology cylinders). This phenomenon was already observed when one replaces 3-manifolds by string-links up to homotopy, see Bar-Natan [B-N] or by string-links up to concordance, see Habegger-Masbaum [HM]. On the other hand, Massey products apply to more general manifolds and they should provide partiallydefined finite-type invariants.

\section{Statement of the RESUlts}

2.1. Conventions. $F$ will always stand for a free group and $H$ for a torsion-free abelian group. The lower central series of a group $G$ is inductively defined by $G_{1}=G$ and $G_{n+1}=\left[G, G_{n}\right]$. A group homomorphism $p: K \rightarrow G$ is called an n-equivalence if it induces an isomorphism $K / K_{n} \cong G / G_{n}$. All manifolds will be oriented, and all maps between them will preserve orientation, unless otherwise mentioned. The boundary of an oriented manifold is oriented with the "outward normal first" convention. 
2.2. Massey products. By Massey products of length $n \geq 2$ in $H^{2}(\pi)$ we mean a Massey product $\left\langle a_{1}, \ldots, a_{n}\right\rangle \in H^{2}(\pi)$ for $a_{i} \in H^{1}(\pi)$, [Ma, FS], which are defined assuming that the ones of length $n-1$ are defined and vanish. We have the following theorem on universal Massey products:

Theorem 1. (i) Given a connected topological space $X$ and 2-equivalence $p: F \rightarrow \pi \stackrel{\text { def }}{=} \pi_{1}(X)$, then $X$ has vanishing Massey products of length less than $n$ if and only if $p$ is an n-equivalence.

(ii) In that case, we have a short exact sequence ${ }^{1}$

$$
H_{2}(X, \mathbb{Z}) \rightarrow \mathrm{L}_{n}\left(H_{1}(X, \mathbb{Z})\right) \rightarrow \pi_{n} / \pi_{n+1} \rightarrow 0,
$$

where the first map determines and is determined by all length $n$ Massey products (for a precise expression, see Corollary 4.3) and the second is induced by the Lie bracket.

(iii) In addition, we have that

$$
\alpha_{1} \smile\left\langle\alpha_{2}, \ldots, \alpha_{n+1}\right\rangle=\left\langle\alpha_{1}, \ldots, a_{n}\right\rangle \smile \alpha_{n+1} \in H^{2}(\pi),
$$

for any $\alpha_{1}, \ldots, \alpha_{n+1} \in H^{1}(\pi)$.

Remark 2.1. For the dependence of the short exact sequence in the above theorem on the map $p$, see Remark 4.4. If $X$ satisfies the hypothesis of Theorem 1 we have dually, over $\mathbb{Q}$ :

$$
0 \rightarrow\left(\pi_{n} / \pi_{n+1}\right)_{\mathbb{Q}}^{*} \rightarrow \mathrm{L}_{n}\left(H^{1}(X, \mathbb{Q})\right) \rightarrow H^{2}(X, \mathbb{Q}) .
$$

Note that the first part of Theorem 1 appears in [O2, Lemma 16], and that the exact sequence above was first suggested by Sullivan in [Su] for $n=2$, and subsequently proven by Lambe in [La] for $n=2$ using different techniques involving minimal models.

Corollary 2.2. Given an n-equivalence $p: F \rightarrow \pi \stackrel{\text { def }}{=} \pi_{1}(M)$, where $M$ is a closed 3-manifold, we have the exact sequence

$$
H^{*} \rightarrow \mathrm{L}_{n}(H) \rightarrow \pi_{n} / \pi_{n+1} \rightarrow 0
$$

where $H=H_{1}(M, \mathbb{Q})$. If $\mu_{n}(M, p) \in H \otimes \mathrm{L}_{n}(H)$ denotes the first map (abbreviated by $\mu_{n}(M)$ if $p$ is clear), then we have that

$$
\mu_{n}(M, p) \in \mathrm{D}_{n}(H) .
$$

In particular, $\mu_{n}(M, p)=0$ if and only if $p: F \rightarrow \pi$ is an $(n+1)$-equivalence.

Given an integer $n$ and a torsion-free abelian group $H$, it is natural to ask which elements of $\mathrm{D}_{n}(H)$ are realized by 3 -manifolds as above. For this see Theorem 4 below.

2.3. The Johnson homomorphism. We now discuss the relation between Massey products and the Johnson homomorphism.

Let $\Gamma_{g, 1}$ denote the mapping class group of a surface $\Sigma_{g, 1}$ of genus $g$ with one boundary component (i.e., the group of surface diffeomorphisms that pointwise preserve the boundary), and let $\Gamma_{g, 1}[n]$ denote its subgroup that consists of surface diffeomorphisms that induce the identity on $\pi / \pi_{n+1}$, where $\pi=\pi_{1}\left(\Sigma_{g, 1}\right)$. In [Jo1], Johnson defined a homomorphism

$$
\tau_{n}: \Gamma_{g, 1}[n] \rightarrow \mathrm{D}_{n+1}(H),
$$

where $H=H_{1}\left(\Sigma_{g, 1}, \mathbb{Z}\right)$, which he further extended to the case of a closed surface. Johnson was well-aware of the relation between his homomorphism and Massey products on mapping torii, i.e., on twisted surface bundles over a circle; see [Jo2, p. 171], further elucidated by Kitano [Ki]. In the present note, we extend this relation to Massey products that come from an arbitrary pair $(\Sigma, M)$ of an imbedding $\iota: \Sigma \hookrightarrow M$ of a closed surface (not necessarily separating) in a 3-manifold. Fix a closed 3-manifold $M$ and an $(n+1)$-equivalence $F \rightarrow \pi \stackrel{\text { def }}{=} \pi_{1}(M)$. Given a pair $(\Sigma, M)$, and $\phi \in \Gamma[n]$, let $M_{\phi}$ denote the result of cutting $M$ along $\Sigma$, twisting by the element $\phi$ of its mapping class group and gluing back. In this case, there exists a canonical cobordism $N_{\phi}$ between $M$ and $M_{\phi}$ such that the maps $\pi_{1}(M) \rightarrow \pi_{1}\left(N_{\phi}\right) \leftarrow \pi_{1}\left(M_{\phi}\right)$ (induced by the inclusions $\left.M, M_{\phi} \hookrightarrow N_{\phi}\right)$ are

\footnotetext{
${ }^{1}$ Note that $\pi_{n}$ denotes the $n$th commutator subgroup of $\pi_{1}(X)$ and not the $n$th homotopy group of $X$.
} 
$(n+1)$-equivalences; thus by Theorem $1, M_{\phi}$ has vanishing Massey products of length less than $n+1$. The ones of length $n+1$ on $M_{\phi}$ are determined in terms of those of $M$ and the Johnson homomorphism as follows:

Theorem 2. With the above assumptions, we have

$$
\mu_{n+1}\left(M_{\phi}\right)=\mu_{n+1}(M)+\iota_{*} \tau_{n}(\phi) .
$$

See also Remark 4.12.

2.4. Homology cylinders and realization. It is well known [Mo3, Ha] that the Johnson homomorphism $\tau_{n}$ is not onto, in other words not every element of $\mathrm{D}_{n+1}(H)$ can be realized by surface diffeomorphisms. Generalizing surface diffeomorphisms to a more general notion of homology cylinders (defined below) allows us to define an ungraded version of the Johnson homomorphism, which then induces, on the associated graded level, generalizations of the Johnson homomorphisms. We will show that all of these are onto, see Theorem 3. As an application of this result, we will show that we can realize every element in $\mathrm{D}_{n}(H)$ by 3 -manifolds as in Corollary 2.2 and, in addition give a proof, free of spectral sequences, of the isomorphism (1), as mentioned above.

Let $\Sigma_{g, 1}$ denote the compact orientable surface of genus $g$ with one boundary component. A homology cylinder over $\Sigma_{g, 1}$ is a compact orientable 3-manifold $M$ equipped with two imbeddings $i^{-}, i^{+}: \Sigma_{g, 1} \rightarrow \partial M$ so that $i^{+}$is orientation-preserving and $i^{-}$is orientation-reversing and if we denote $\Sigma^{ \pm}=\operatorname{Im} i^{ \pm}\left(\Sigma_{g, 1}\right)$, then $\partial M=\Sigma^{+} \cup \Sigma^{-}$and $\Sigma^{+} \cap \Sigma^{-}=\partial \Sigma^{+}=\partial \Sigma^{-}$. We also require that $i^{ \pm}$be homology isomorphisms. We can multiply two homology cylinders by identifying $\Sigma^{-}$in the first with $\Sigma^{+}$in the second via the appropriate $i^{ \pm}$. Thus $\mathcal{H}_{g, 1}$, the set of orientation-preserving diffeomorphism classes of homology cylinders over $\Sigma_{g, 1}$ is a semi-group with an obvious identity.

There is a canonical homomorphism $\Gamma_{g, 1} \rightarrow \mathcal{H}_{g, 1}$ that sends $\phi$ to $\left(I \times \Sigma_{g, 1}, 0 \times\right.$ id, $\left.1 \times \phi\right)$. Nielsen showed that the natural map $\Gamma_{g, 1} \rightarrow \mathrm{A}_{0}(F)$ is an isomorphism, where $F$ is the free group on $2 g$ generators $\left\{x_{i}, y_{i}\right\}$, identified with the fundamental group of $\Sigma_{g, 1}$ (with base-point on $\partial \Sigma_{g, 1}$ ), and $\mathrm{A}_{0}(F)$ is the group of automorphisms of $F$ which fix the element $\omega_{g}=\left[x_{1}, y_{1}\right] \cdots\left[x_{g}, y_{g}\right]$, representing the boundary of $\Sigma_{g, 1}$. It is natural to ask whether there exists an analogous isomorphism for the semigroup $\mathcal{H}_{g, 1}$. Below, we construct for every $n$ a homomorphism $\sigma_{n}: \mathcal{H}_{g, 1} \rightarrow \mathrm{A}_{0}\left(F / F_{n}\right)$, where $\mathrm{A}_{0}\left(F / F_{n}\right)$ is the group of automorphisms $\phi$ of $F / F_{n}$ such that a lift of $\phi$ to an endomorphism $\bar{\phi}$ of $F$ fixes $\omega_{g} \bmod F_{n+1}$. It is easy to see that this condition is independent of the lift. For example $A_{0}\left(F / F_{2}\right)=\operatorname{Sp}(g, \mathbb{Z})$.

Given $\left(M, i^{+}, i^{-}\right) \in \mathcal{H}_{g, 1}$ consider the homomorphisms $i_{*}^{ \pm}: F \rightarrow \pi_{1}(M)$, where the base-point is taken in $\partial \Sigma^{+}=\partial \Sigma^{-}$. In general, $i_{*}^{ \pm}$are not isomorphisms- however, since $i^{ \pm}$are homology isomorphisms, it follows from Stallings [St] that they induce isomorphisms $i_{n}^{ \pm}: F / F_{n} \rightarrow \pi_{1}(M) / \pi_{1}(M)_{n}$. We then define $\sigma_{n}\left(M, i^{ \pm}\right)=\left(i_{n}^{-}\right)^{-1} \circ i_{n}^{+}$. It is easy to see that $\sigma_{n}\left(M, i^{ \pm}\right) \in \mathrm{A}_{0}\left(F / F_{n}\right)$.

Theorem 3. The map $\sigma_{n}: \mathcal{H}_{g, 1} \rightarrow \mathrm{A}_{0}\left(F / F_{n}\right)$ is surjective.

Remark 2.3. We can convert $\mathcal{H}_{g, 1}$ into a group $\mathcal{H}_{g, 1}^{c}$ by considering homology cobordism classes of homology cylinders. The inverse of an element is just the reflection in the $I$ coordinate. It is easy to see that the invariants $\sigma_{n}$ just depend on the homology bordism class and so define homomorphisms $\mathcal{H}_{g, 1}^{c} \rightarrow \mathrm{A}_{0}\left(F / F_{n}\right)$. The natural homomorphism $\Gamma_{g, 1} \rightarrow \mathcal{H}_{g, 1}^{c}$ is seen to be injective by the existence of the $\sigma_{n}$ and the fact that the homomorphism $\Gamma_{g, 1} \rightarrow \mathrm{A}_{0}(F)$ is an isomorphism.

In addition, we can combine the maps $\sigma_{n}$, for all $n$, to a single map $\sigma^{\text {nil }}: \mathcal{H}_{g, 1} \rightarrow \mathrm{A}_{0}\left(F^{\text {nil }}\right)$, where $F^{\text {nil }}$ is the nilpotent completion of $F$. Unlike $\sigma, \sigma^{\text {nil }}$ is not one-to-one, i.e., $\cap_{n} \operatorname{Ker} \sigma_{n} \neq\{1\}$. For example, if $P$ is any homology sphere, then the connected sum $\left(I \times \Sigma_{g, 1}\right) \sharp P$ defines an element in the kernel. Also $\sigma^{\text {nil }}$ is not onto, even though each $\sigma_{n}$ is. To identify the image of $\sigma^{\text {nil }}$ we have to consider the algebraic closure $\bar{F} \subseteq F^{\text {nil }}$, see [Le2]. Using the arguments of [Le2], we can show that any element of $\operatorname{Im}\left(\sigma^{\text {nil }}\right)$ restricts to an automorphism of $\bar{F}$ and, by arguments similar to the proof of Theorem 3, it can be proved that $\operatorname{Im}\left(\sigma^{\text {nil }}\right)$ consists precisely of those $\phi \in A_{0}\left(F^{\text {nil }}\right)$ which restrict 
to an automorphism of $\bar{F}$ and such that the element of $H_{2}(\bar{F})$ associated to $\phi$ (see the proof of Theorem 3) is zero. But since we do not know whether $H_{2}(\bar{F})=0$, this result does not seem very useful at this time.

Remark 2.4. The $\left\{\sigma_{n}\right\}$ can be described by numerical invariants if we consider the coefficients of the Magnus expansion of $\sigma_{n}(M)\left(x_{i}\right), \sigma_{n}(M)\left(y_{i}\right)$. This is analogous to the definition of the $\mu$-invariants of a string link. We can refer to these as $\mu$-invariants of homology cylinders.

It will be useful for us to consider the filtration defined by the maps $\sigma_{n}$, namely we define a decreasing weight filtration on $\mathcal{H}_{g, 1}$ and on $\mathcal{H}_{g, 1}^{c}$ by setting $\mathcal{H}_{g, 1}[n]=\operatorname{Ker}\left(\sigma_{n}\right)$.

Proposition 2.5. We have an exact sequence

$$
1 \rightarrow \mathrm{D}_{n}(H) \rightarrow \mathrm{A}_{0}\left(F / F_{n+1}\right) \rightarrow \mathrm{A}_{0}\left(F / F_{n}\right) \rightarrow 1
$$

and a commutative diagram

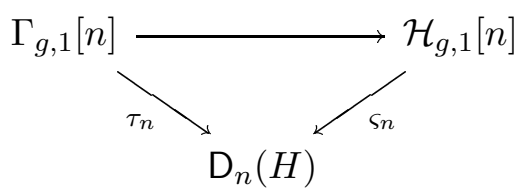

where the map $\varsigma_{n}$, induced by $\sigma_{n}$, is onto. It follows that

$$
0 \rightarrow \mathcal{H}_{g, 1}^{c}[n+1] \rightarrow \mathcal{H}_{g, 1}^{c}[n] \stackrel{\varsigma_{n}}{\rightarrow} \mathrm{D}_{n}(H) \rightarrow 0
$$

is exact.

Remark 2.6. A major problem in the study of the mapping class group is to determine the image of the Johnson homomorphism $\tau_{n}$, which largely determines the algebraic structure of the mapping class group since $\cap_{n} \Gamma_{g, 1}[n]=1$. In contrast, Theorem 3 largely determines the structure of $\mathcal{H}_{g, 1} / \mathcal{H}_{g, 1}[\infty]$, but in this case $\mathcal{H}_{g, 1}[\infty]=\cap_{n} \mathcal{H}_{g, 1}[n]$ is not trivial - see Question 7 at the end of the paper.

Remark 2.7. It is instructive to consider the analogy between, on the one hand, the mapping class group, homology cylinders and the invariant $\sigma_{n}$ and the Johnson homomorphism, and, on the other hand, the pure braid group, string links and the Milnor $\mu$-invariants. There is an injection of the pure braid group on $g$ strands into the mapping class group $\Gamma_{g, 1}$, first defined by Oda and studied in [Le2], which preserves the weight filtrations and induces a monomorphism of the associated graded Lie algebras. This can, in fact, be generalized to an injection of the semi-group $\mathcal{S}_{g}$ of string links on $g$ strands into the semi-group $\mathcal{H}_{g, 1}$ (and of the string-link concordance group $\mathcal{S}_{g}^{c}$ into $\mathcal{H}_{g, 1}^{c}$ ), under which $\sigma_{n}$ and the $\mu$-invariants correspond. We will explain this in a future paper.

Theorem 4. Every element in $\mathrm{D}_{n}(H)$ is realized by an $n$-equivalence $F \rightarrow \pi_{1}\left(M^{3}\right)$, for some closed 3-manifold $M$, as in Corollary 2.2. In addition, a 2-equivalence $F \rightarrow H$ gives rise to a map $H_{3}\left(F / F_{n}\right) \rightarrow H \otimes \mathrm{L}_{n}(H)$ inducing the isomorphism of Equation (1).

We will give two different proofs of this theorem. One approach is to apply results of Orr [O1] and Igusa-Orr [IO] on $H_{3}\left(F / F_{n}\right)$ and, in particular, the isomorphism

$$
\operatorname{cok}\left(H_{3}\left(F / F_{n+1}\right) \rightarrow H_{3}\left(F / F_{n}\right)\right) \cong \mathrm{D}_{n}(H) .
$$

A very similar argument appears in [CGO].

Alternatively we will see that this realizability is a consequence of Theorem 3. This approach has the advantage of being "spectral-sequence-free" and also gives another proof of (1).

2.5. Homology cylinders and finite type invariants of 3-manifolds. Goussarov and Habiro [Gu1, Gu2, Hb] have studied two rather dual notions: an $n$-equivalence relation among 3-manifolds, and a theory of invariants of 3-manifolds with values in an abelian group. Since their work is recent and not yet fully written, we will, for the benefit of the reader, give a short introduction using terminology and notation from [GGP] (to which we refer the reader for detailed proofs). Both 
notions are intimately related to that of surgery $M_{\Gamma}$ along a $Y$-link $\Gamma$ in a 3 -manifold $M$, i.e., surgery along an imbedded link associated to an imbedding of an appropriately oriented, framed graph with trivalent and univalent vertices so that the univalent ones end in "leaves" (explained below). Two manifolds are n-equivalent if one can pass from one to the other by surgery on a $Y$-link associated to a connected Y-graph of degree (i.e., number of trivalent vertices) at least $n$. For example a theorem of Matveev [M] says that two closed manifolds are 1-equivalent if and only if there is an isomorphism between their first homology groups which preserves the linking form on the torsion subgroups. Similarly, a finite type invariant $\lambda$ ought to be the analog of a polynomial on the set of 3-manifolds, in other words for some integer $n$ it satisfies a difference equation

$$
\sum_{\Gamma^{\prime} \subseteq \Gamma}(-1)^{\left|\Gamma^{\prime}\right|} \lambda\left(M_{\Gamma}\right)=0
$$

where $\Gamma$ is a $Y$-link in $M$ of more than $n$ components and the sum is over all $Y$-sublinks $\Gamma^{\prime}$ of $\Gamma$. In view of the above definition, it is natural to consider the free abelian group $\mathcal{M}$ generated by homeomorphism classes of closed oriented 3-manifolds, and to define a decreasing filtration $\mathcal{F}^{Y} \mathcal{M}$ on $\mathcal{M}$ in such a way that $\lambda$ is an invariant of type $n$ if and only if it vanishes on $\mathcal{F}_{n+1}^{Y} \mathcal{M}$. Thus the question of how many invariants of degree $n$ there are translates into a question about the size of the graded quotients $\mathcal{G}_{n}^{Y} \mathcal{M} \stackrel{\text { def }}{=} \mathcal{F}_{n}^{Y} \mathcal{M} / \mathcal{F}_{n+1}^{Y} \mathcal{M}$. One traditionally approaches this problem by giving independently an upper bound and a lower bound, which hopefully match. In this theory, an upper bound has been obtained in terms of an abelian group of decorated graphs as follows. One observes first that surgery along $Y$-links preserves the homology and linking form of 3-manifolds, as well as the boundary. Define an equivalence relation on compact 3-manifolds: $M \sim N$ if there exists an isomorphism $\rho: H_{1}(M) \rightarrow H_{1}(N)$ inducing an isometry of the linking forms, and a homeomorphism $\partial M \rightarrow \partial N$ consistent with $\rho$. Thus if we let $\mathcal{M}(M)$ denote the subgroup of $\mathcal{M}$ generated by equivalent 3-manifolds, we have a direct sum decomposition $\mathcal{M}=\oplus_{\sim} \mathcal{M}(M)$ (and also, $\mathcal{F}^{Y} \mathcal{M}=\oplus \sim \mathcal{F}^{Y} \mathcal{M}(M)$ ), where the sum is over a choice of one manifold $M$ from each equivalence class. In fact for closed 3-manifolds Matveev's theorem tells us that $\mathcal{G}_{0}^{Y} \mathcal{M}(M)=\mathbb{Z}$. After we fix a 3 -manifold $M$, and an oriented link $\mathfrak{b}$ in $M$ that represents a basis of $H_{1}(M, \mathbb{Z}) /$ torsion, together with a framing of $\mathfrak{b}$ (i.e., a choice of a trivialization of the normal bundle of each component of $\mathfrak{b})$, it turns out that there is a map ${ }^{2}$

$$
W_{n}^{\mathfrak{b}}: \mathcal{A}_{n}(M) \rightarrow \mathcal{G}_{n}^{Y} \mathcal{M}(M),
$$

which is onto over $\mathbb{Q}$ (actually, onto over $\mathbb{Z}[1 /(2 \mid$ torsion $\mid)]$ ), where $\mathcal{A}(M)$ is the group generated by graphs with univalent and trivalent vertices, with a cyclic order along each trivalent vertex, decorated by an element of $H_{1}(M, \mathbb{Z})$ on each univalent vertex, modulo some relations, see [GGP]. Here $\mathcal{A}_{n}(M)$ is the subgroup generated by graphs of degree $n$, i.e., with $n$ trivalent vertices; thus we have $\mathcal{A}(M)=\oplus_{n} \mathcal{A}_{n}(M)=\mathcal{A}^{t}(M) \oplus \mathcal{A}^{l}(M)$, where $\mathcal{A}^{t}(M)$ (resp. $\mathcal{A}^{l}(M)$ ) is the subgroup of $\mathcal{A}(M)$ generated by trees (resp. graphs with nontrivial first homology). For a detailed discussion of the map $W^{\mathfrak{b}}$, see also Section 3 .

We should point out that for $M=S^{3}$ (i.e., for integral homology 3-spheres) one can construct sufficiently many invariants of integral homology 3 -spheres to show that $W^{\mathfrak{b}}$ is an isomorphism, over $\mathbb{Q}$, see $[\mathrm{LMO}]$. The same is true for finite type (i.e., Vassiliev) invariants of links in $S^{3}$, over $\mathbb{Q}$, see $[\mathrm{Ko} 2]$. However, it is at present unknown whether the map (2) is one-to-one (and thus, an isomorphism), over $\mathbb{Q}$, for all 3-manifolds.

We now discuss a well-known isomorphism $[\mathrm{Ih}, \mathrm{O} 2, \mathrm{Dr}, \mathrm{HM}]$, over $\mathbb{Q}$, for a torsion-free abelian group $A$ :

$$
\Psi_{n}: \mathcal{A}_{n}^{t}(A) \cong_{\mathbb{Q}} \mathrm{D}_{n+1}(A),
$$

\footnotetext{
2 a more precise notation, which we will not use, would be $W_{n}^{M, \mathfrak{b}}$.
} 
which will help us relate the Johnson homomorphism to the tree-level part of finite type invariants of 3-manifolds. This map is defined as follows: Fix an oriented uni-trivalent tree $T$ of degree $n$ (thus with $n+2$ legs, i.e., univalent vertices) and let $c: \operatorname{Leg}(T) \rightarrow A$ be a coloring of its legs. Given a leg $l$ of $T,(T, l)$ is a rooted colored tree to which we can associate an element $(T, l)$ of $\mathrm{L}_{n+1}(A)$. Due to the $I H X$ relation (see Figure 1), the function

$$
T \rightarrow \sum_{l \in \operatorname{Leg}(T)} c(l) \otimes(T, l)
$$

descends to one $\mathcal{A}_{n}^{t}(A) \rightarrow A \otimes \mathrm{L}_{n+1}(A)$ so that its composition with $A \otimes \mathrm{L}_{n+1}(A) \rightarrow \mathrm{L}_{n+2}(A)$ vanishes, thus defining the map $\Psi_{n}$. There is a map $A \otimes \mathrm{L}_{n+1}(A) \rightarrow \mathcal{A}_{n}^{t}(A)$ (defined by sending $a \otimes b \in A \otimes \mathrm{L}_{n+1}(A)$ to the rooted tree with one root colored by $a$ and $n$ additional legs colored by $c$ ), which shows that $\Psi_{n}$ is one-to-one; and by counting ranks it follows that it is in fact a vector space isomorphism. It is unknown to the authors whether $\Psi_{n}$ is an isomorphism over $\mathbb{Z}[1 / 6]$.

$$
\underset{c}{*} \gtrless_{b}^{a} \longrightarrow[c,[a, b]],{\underset{c}{f}}^{b} \longrightarrow a \otimes[c, b]+c \otimes[b, a]+b \otimes[a, c]
$$

Figure 1. On the left, the map from rooted vertex-oriented trees to the free Lie algebra; on the right the map $\Psi_{1}$.

It turns out that a skew-symmetric form $\mathfrak{c}: A \otimes A \rightarrow \mathbb{Q}$ equips $\mathcal{A}^{t}(A)$ with the structure of a graded Lie algebra, by defining the Lie bracket

$$
\left[\Gamma, \Gamma^{\prime}\right]^{\mathfrak{c}}=\sum_{a, b} \mathfrak{c}(a, b)\left(\Gamma_{a} \text { glue } \Gamma_{b}^{\prime}\right)
$$

where the sum is over each leg $a$ of $\Gamma$ and $b$ of $\Gamma^{\prime}$ and $\Gamma_{a}$ glue $\Gamma_{b}^{\prime}$ is the graph obtained by gluing the legs $a$ and $b$ of $\Gamma$ and $\Gamma^{\prime}$ respectively, with the understanding that the sum over an empty set is zero. In other words, $\left[\Gamma, \Gamma^{\prime}\right]^{\mathfrak{c}}$ is the sum of all contractions of a leg of $\Gamma$ with a leg of $\Gamma^{\prime}$. This Lie bracket is not new, it has been observed and used by Morita [Mo2] and Kontsevich [Ko1] on a close relative of $\mathcal{A}^{t}(A)$, namely $\mathrm{D}^{\prime}(A) \stackrel{\text { def }}{=} A^{*} \otimes \mathrm{L}(A)$ (which carries a bracket of degree -1 ).

We now explain the Lie bracket on $\mathcal{A}^{t}(A)$ from the point of view of finite type invariants of 3-manifolds. Fixing a compact surface $\Sigma_{g, 1}$ of genus $g$ with one boundary component, it follows by definition that $\mathcal{M}\left(\Sigma_{g, 1} \times I\right)$ is generated by homology cylinders over $\Sigma_{g, 1}$ and is a ring with multiplication $M_{1} * M_{2}$ defined by stacking $M_{1}$ below $M_{2}$. Fix a framed oriented link $\mathfrak{b}$ in $\Sigma_{g, 1} \times I$ that represents a basis of $H_{1}\left(\Sigma_{g, 1} \times I ; \mathbb{Z}\right)$ and consider the associated onto map $W^{\mathfrak{b}}: \mathcal{A}\left(\Sigma_{g, 1} \times I\right) \rightarrow$ $\mathcal{G}^{Y} \mathcal{M}\left(\Sigma_{g, 1} \times I\right)$ from (2), which is expected to be an isomorphism. Thus, $\mathcal{A}\left(\Sigma_{g, 1} \times I\right)$ should be equipped with a ring structure. This is the content of the following

Proposition 2.8. (i) $\mathfrak{b}$ induces a homomorphism $\langle\cdot, \cdot\rangle^{\mathfrak{b}}: H_{1}\left(\Sigma_{g, 1}, \mathbb{Z}\right) \otimes H_{1}\left(\Sigma_{g, 1}, \mathbb{Z}\right) \rightarrow \mathbb{Z}$ satisfying ${ }^{3}$

$$
\langle a, b\rangle^{\mathfrak{b}}-\langle b, a\rangle^{\mathfrak{b}}=a \cdot b,
$$

where $\cdot$ is the natural symplectic form on $H_{1}\left(\Sigma_{g, 1}, \mathbb{Z}\right)$.

(ii) $\mathcal{A}\left(\Sigma_{g, 1} \times I\right)$ is a ring with $*$-multiplication (depending on $\mathfrak{b}$ ) defined as follows: for $\Gamma, \Gamma^{\prime} \in$ $\mathcal{A}\left(\Sigma_{g, 1} \times I\right)$,

$$
\Gamma * \Gamma^{\prime}=\sum_{l=0}^{\infty}\left\langle\Gamma, \Gamma^{\prime}\right\rangle_{l}
$$

\footnotetext{
${ }^{3}\langle\cdot, \cdot\rangle^{\mathfrak{b}}$ will often be denoted by $\langle\cdot, \cdot\rangle$ if $\mathfrak{b}$ is clear from the context.
} 
where

$$
\left\langle\Gamma, \Gamma^{\prime}\right\rangle_{l}=(-1)^{l} \sum_{a, b} \prod_{i=1}^{l}\left\langle a_{i}, b_{i}\right\rangle^{\mathfrak{b}}\left(\Gamma_{a} \text { glue } \Gamma_{b}^{\prime}\right)
$$

is the sum over all ordered subsets $a=\left(a_{1}, \ldots, a_{l}\right)$ and $b=\left(b_{1}, \ldots, b_{l}\right)$ of the set of legs of $\Gamma$ and $\Gamma^{\prime}$ respectively, $\Gamma_{a}$ glue $\Gamma_{b}^{\prime}$ is the graph obtained by gluing the $a_{i}$-leg of $\Gamma$ to the $b_{i}$-leg of $\Gamma^{\prime}$, for every $i$, with the understanding that a sum over the empty set is zero (thus the multiplication $*$ is a finite sum).

(iii) $\mathcal{A}^{c}\left(\Sigma_{g, 1} \times I\right)$ is a Lie subring of $\mathcal{A}\left(\Sigma_{g, 1} \times I\right)$ with bracket defined by

$$
\left[\Gamma, \Gamma^{\prime}\right]=\Gamma * \Gamma^{\prime}-\Gamma^{\prime} * \Gamma=\sum_{l=1}^{\infty}\left\langle\Gamma, \Gamma^{\prime}\right\rangle_{l}-\left\langle\Gamma^{\prime}, \Gamma\right\rangle_{l}
$$

(iv) Over $\mathbb{Q}$, there is an algebra isomorphism $\mathrm{U}\left(\mathcal{A}^{c}\left(\Sigma_{g, 1} \times I\right)\right) \cong_{\mathbb{Q}} \mathcal{A}\left(\Sigma_{g, 1} \times I\right)$, where $\mathrm{U}$ is the universal enveloping algebra functor.

Remark 2.9. The leading term $\langle\cdot, \cdot\rangle_{1}$ of the $*$-multiplication that involves contracting a single leg is independent of $\mathfrak{b}$ (see also part (iii) of Theorem 5 ), whereas the subleading terms $\langle\cdot, \cdot\rangle_{l}$ for $l \geq 2$ depend on $\mathfrak{b}$. This is a common phenomenon in mathematical physics, analogous to the fact that differential operators such as the Laplacian or the Dirac depend on a Riemannian metric, but have symbols independent of it.

Remark 2.10. It is interesting to compare $\langle a, b\rangle^{\mathfrak{b}}$ to the Seifert matrix of a knot. Both notions depend on "linking numbers" of "stacked" curves, i.e. curves pushed in a positive or negative direction and the relation of $\langle\cdot, \cdot\rangle^{\mathfrak{b}}$ and the Seifert matrix to the symplectic structure on $H_{1}(\Sigma)$ is the same. The noncommutativity of the stacking is reflected by the fact that the form $\langle\cdot, \cdot\rangle^{\mathfrak{b}}$ is not symmetric. For a related appearance of this noncommutativity, see also [AMR1, AMR2]. Over $\mathbb{Q}$, the Lie algebra $\mathcal{A}^{c}\left(\Sigma_{g, 1} \times I\right)$ is closely related to a Lie algebra of chord diagrams on $\Sigma$ considered by Andersen-Mattes-Reshetikhin in relation to deformation quantization, loc. cit. We will postpone an explanation of this relation to a subsequent publication.

The ring structure on $\mathcal{A}\left(\Sigma_{g, 1} \times I\right)$ would be of little interest were it not compatible with the one of $\mathcal{G}^{Y} \mathcal{M}\left(\Sigma_{g, 1} \times I\right)$ and $\mathcal{A}^{t}\left(\Sigma_{g, 1} \times I\right)$; this is the content of the following

Theorem 5. (i) The map $W^{\mathfrak{b}}: \mathcal{A}\left(\Sigma_{g, 1} \times I\right) \rightarrow \mathcal{G}^{Y} \mathcal{M}\left(\Sigma_{g, 1} \times I\right)$ preserves the ring structure. (ii) $\mathcal{A}^{l}\left(\Sigma_{g, 1} \times I\right)$ is a Lie ideal of $\mathcal{A}^{c}\left(\Sigma_{g, 1} \times I\right)$.

(iii) The Lie bracket of the quotient $\mathcal{A}^{c}(\Sigma \times I) / \mathcal{A}^{l}\left(\Sigma_{g, 1} \times I\right) \cong \mathcal{A}^{t}\left(\Sigma_{g, 1} \times I\right)$ is equal to $(-1)^{\operatorname{deg}-1}$ times the Lie bracket of Equation (4) using the symplectic form on $H_{1}\left(\Sigma_{g, 1} \times I\right) \cong H_{1}\left(\Sigma_{g, 1}\right)$. In particular, it is independent of the basis $\mathfrak{b}$.

From now on, we will work over $\mathbb{Q}$. We now show that the Johnson homomorphism $\tau: \mathcal{G} \mathcal{T}_{g, 1} \rightarrow$ $\mathcal{A}^{t}\left(\Sigma_{g, 1} \times I\right)$, or rather its signed version $\bar{\tau} \stackrel{\text { def }}{=}(-1)^{\operatorname{deg}-1} \tau$, can be recovered from the Lie algebra structure on $\mathcal{A}^{c}\left(\Sigma_{g, 1} \times I\right)$, where $\mathcal{T}_{g, 1}(n) \subseteq \Gamma_{g, 1}[n]$ is the subgroup of the Torelli group generated by $n$-fold commutators, and $\mathcal{G}_{n} \mathcal{T}_{g, 1}$ denotes the quotient $\mathcal{T}_{g, 1}(n) / \mathcal{T}_{g, 1}(n+1)$. Recall the map $\mathcal{T}_{g, 1} \rightarrow$ $\mathcal{M}\left(\Sigma_{g, 1} \times I\right)$ defined by changing the parametrization of the top part of homology cylinders, its linear extension $\left(I \mathcal{T}_{g, 1}\right)^{n} \rightarrow \mathcal{F}_{n}^{Y} \mathcal{M}\left(\Sigma_{g, 1} \times I\right)$, where $I \mathcal{T}_{g, 1}$ is the augmentation ideal of the group ring $\mathbb{Q} \Gamma_{g, 1}$, and the induced algebra map $\mathcal{G} \mathcal{T}_{g, 1} \rightarrow \mathcal{G}^{Y} \mathcal{M}\left(\Sigma_{g, 1} \times I\right)$. The theorem below explains the statement that the Johnson homomorphism is contained in the tree-level part of a theory of invariants in $\Sigma_{g, 1} \times I$. 
Theorem 6. Given a surface $\Sigma_{g, 1}$ as above of genus at least 6 , there exists a map $\Phi: \mathcal{G T}_{g, 1} \rightarrow$ $\mathcal{A}^{c}\left(\Sigma_{g, 1} \times I\right)$ and commutative diagrams of graded Lie algebras:

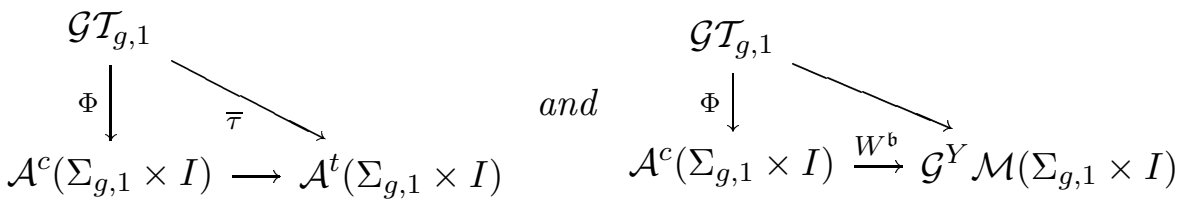

where the left horizontal map is the natural projection on the tree part. In other words, for $\phi \in$ $\mathcal{T}_{g, 1}(n)$ we have

in $\mathcal{A}^{c}\left(\Sigma_{g, 1} \times I\right)$.

$$
\Phi_{n}(\phi)=(-1)^{n-1} \tau_{n}(\phi)+\text { loops }
$$

Remark 2.11. For a closed surface $\Sigma_{g}$ of genus $g$, there is an identical version of Proposition 2.8 and Theorem 5 above. As for Theorem 6 , given a closed surface $\Sigma_{g}$ of genus at least 6 , there exists commutative diagrams

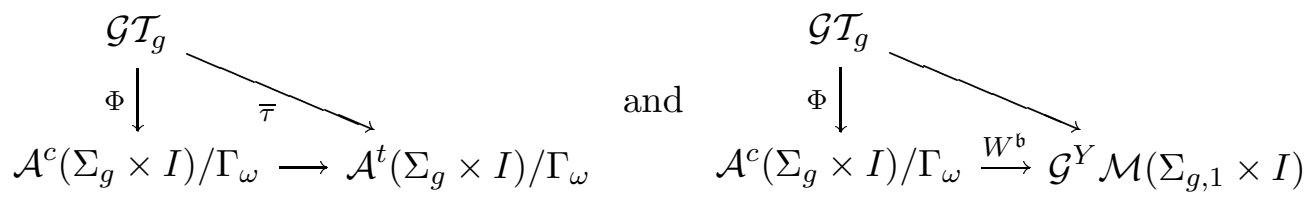

where $\Gamma_{\omega}$ is the ideal of $\mathcal{A}\left(\Sigma_{g} \times I\right)$ which is generated by all elements of the form $\sum_{i} \Gamma_{x_{i}, y_{i}, a}$ where $\left\{x_{i}, y_{i}\right\}$ is a standard symplectic basis of $H_{1}\left(\Sigma_{g}\right), a \in H_{1}\left(\Sigma_{g}\right)$ and $\Gamma_{b, c, d}$ denote the degree 1 graph

$$
{ }^{b} Y^{c}
$$

with counterclockwise orientation.

It is natural to ask for a statement of the above theorem involving general (closed) 3-manifolds $M$. How does one construct elements in $\mathcal{F}^{Y} \mathcal{M}(M)$ ? Given an imbedding $\iota: \Sigma \hookrightarrow M$ of a closed surface $\Sigma$ and $\phi \in \mathcal{T}(n)$, it was shown in [GGP] that $M-M_{\phi} \in \mathcal{F}_{n}^{Y} \mathcal{M}(M)$. The following theorem relates the Johnson homomorphism and the tree-level part of finite type invariants of 3-manifolds.

Theorem 7. With the above assumptions, we can find $c_{\phi, n}^{\mathfrak{b}} \in \mathcal{A}_{n}^{l}(M)$ so that we have in $\mathcal{G}_{n}^{Y} \mathcal{M}(M)$ :

$$
W_{n}^{\mathfrak{b}}\left(\Psi_{n}^{-1} \iota_{*} \bar{\tau}_{n}(\phi)+c_{\phi, n}^{\mathfrak{b}}\right)=M-M_{\phi} .
$$

The above theorem should be compared with [HM, Theorem 6.1], where they show that if a string link $L$ has vanishing $\mu$-invariants of length less than $n$, then the degree $n$ tree-level part of the Kontsevich integral of $L$ is given by the degree $n \mu$-invariants of $L$. Note that these $\mu$-invariants are Massey products on the closed 3-manifold obtained by 0-surgery along the closure of the string link.

2.6. Plan of the proof. The paper consists of two, largely independent sections; the reader could easily skip one of them without any loss of understanding of the results of the other. Two notions that jointly appear in Sections 3 and 4 are the Johnson homomorphism and the notion of homology cylinders.

In Section 3, we use combinatorial techniques that are usually grouped under the name of finite type invariants (of knotted objects such as braids, links, string links or 3-manifolds) or graph cohomology. A key aspect is the introduction of a Lie algebra $\mathcal{A}\left(\Sigma_{g, 1} \times I\right)$ of graphs and its relation to the Johnson homomorphism, via Theorems 2.8, 5, 6 and 7 .

In Section 4, we use standard techniques from algebraic and geometric topology to prove Theorems 1 concerning Massey products in general spaces and closed 3-manifolds,in particular, and Theorem 2 which relates the Johnson homomorphism to Massey products. In addition, we use 
standard surgery techniques adapted to homology cylinders to prove the two realization Theorems 3 and 4.

Finally, in Section 5 we pose a set of questions that naturally arise in our present study.

\section{Finite type inVARIANTS OF 3 -MANifoldS}

This section concentrates on the proof of Theorems 2.8, 5, 6 and 7 . The techniques that we use are a combination of geometric and combinatorial arguments.

Proof. (of Proposition 2.8) We only explain the first part. Statements (ii) and (iii) are obvious and (iv) follows by a theorem of Milnor-Moore $[\mathrm{MM}]$ regarding the structure of cocommutative graded connected Hopf algebras.

First we arrange that the components of $\mathfrak{b}$ project to immersions in $\Sigma$ with transverse selfintersections and so that the framing has its first componenet vector field pointing in the $I$ direction. We call such a link generic. Given a two-component sublink $\left\{b_{1}, b_{2}\right\}$ of the framed oriented link $\mathfrak{b}$ in $\Sigma_{g, 1} \times I$, let $\left\{p\left(b_{1}\right), p\left(b_{2}\right)\right\}$ denote its projection on $\Sigma \times 0$. Then $p\left(b_{1}\right)$ and $p\left(b_{2}\right)$ intersect transversely at double points. Define $\left\langle b_{1}, b_{2}\right\rangle^{\mathfrak{b}}$ to be the sum with signs over all points in $p\left(b_{1}\right) \cap p\left(b_{2}\right)$ that $p\left(b_{1}\right)$ overcrosses $p\left(b_{2}\right)$, according to the convention

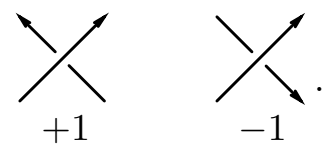

If $b_{1}=b_{2}$, then we define $\left\langle b_{1}, b_{1}\right\rangle^{\mathfrak{b}}$ by counting the self-intersections of $b_{1}$ in the above manner. Since $\mathfrak{b}$ is a basis of $H_{1}\left(\Sigma_{g, 1} \times I, \mathbb{Z}\right) \cong H_{1}(\Sigma, \mathbb{Z})$, this defines, by linearity, the desired map $\langle\cdot, \cdot\rangle^{\mathfrak{b}}$.

Since $p\left(b_{1}\right) \cdot p\left(b_{2}\right)$ is the sum with signs over all points $p\left(b_{1}\right) \cap p\left(b_{2}\right)$, it follows that $\langle a, b\rangle-\langle b, a\rangle=$ $a \cdot b$ for $a, b \in H_{1}(\Sigma, \mathbb{Z})$.

Before we proceed with the proof of Theorems 5 and 7 , we need to recall the definition of the map $W^{\mathfrak{b}}: \mathcal{A}(M) \rightarrow \mathcal{G}^{Y} \mathcal{M}(M)$ : Given a colored graph $\Gamma$, we will construct an imbedding of it in $M$ in two steps.

First, we imbed the leaves, as follows. Given the decoration $x \in H_{1}(M, \mathbb{Z})$, consider its projection $x^{t f} \in H_{1}(M, \mathbb{Z}) /$ torsion and write $x^{t f}=\sum_{b \in \mathfrak{b}} n_{b}[b]$, for integers $n_{b}$. Consider the oriented link $L_{x}$ obtained by the union (over $b$ ) of $n_{b}$ parallel copies of $b$, where parallel copies of a component of $\mathfrak{b}$ are obtained by pushing off using the framing of $\mathfrak{b}$. Choose a basing for $\mathfrak{b}$, i.e., a set or meridians on each component of $\mathfrak{b}$ together with a path to a base point. Join the components of $L_{x}$ using this basing to construct a knot $K_{x}$ in $M$. Apply this construction to every leaf of $\Gamma$. Of course, the resulting link depends on the above choices of basing and joining.

Second, imbed the edges of $\Gamma$ arbitrarily in $M$.

This defines an imbedding of $\Gamma$ in $M$ (which we denote by the same name) that also depends on the above choices; however the associated element $[M, \Gamma]$ in $\mathcal{G}^{Y} \mathcal{M}(M)$, where

$$
[M, \Gamma]=\sum_{\Gamma^{\prime} \subseteq \Gamma}(-1)^{\left|\Gamma^{\prime}\right|} M_{\Gamma^{\prime}}
$$

is the alternating sum over all $Y$-sublinks $\Gamma^{\prime}$ of $\Gamma$, is well-defined, depending only on the framed link $\mathfrak{b}$. This follows from the following equalities in $\mathcal{G}^{Y} \mathcal{M}(M)$ (for detailed proofs see [Gu2, Hb] and also [GGP]):

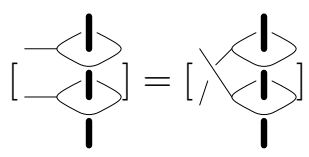



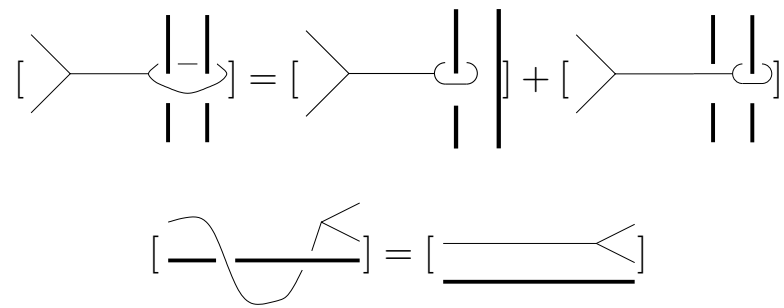

where in the above equalities $[M, \Gamma]$ is abbreviated by $[\Gamma]$. Using further identities in $\mathcal{G}^{Y} \mathcal{M}(M)$, one can show loc.cit. that the map $\Gamma \rightarrow[M, \Gamma]$ factors through further relations to define a map $W^{\mathfrak{b}}: \mathcal{A}(M) \rightarrow \mathcal{G}^{Y} \mathcal{M}(M)$.

Proof. (of Theorem 5) For the first part of the theorem, we begin by choosing $\mathfrak{b}$ in $\Sigma_{g, 1} \times I$ to be a generic link. Note that $\mathfrak{b}$ can be recovered from its projection $p(\mathfrak{b})$ together with a knowledge of the signs at each overcrossing.

Given $\Gamma \in \mathcal{A}\left(\Sigma_{g, 1} \times I\right)$, let $L^{\mathfrak{b}}(\Gamma)$ be an associated $Y$-link in $\Sigma_{g, 1} \times I$ such that $W^{\mathfrak{b}}(\Gamma)=$ $\left[\Sigma_{g, 1} \times I, L^{\mathfrak{b}}(\Gamma)\right]$. Without loss of generality, we can assume that the leaves $l^{\mathfrak{b}}(\Gamma)$ of $L^{\mathfrak{b}}(\Gamma)$ form a generic link, and by abuse of notation, we can write that $W^{\mathfrak{b}}(\Gamma)=\left[\Sigma_{g, 1} \times I, p\left(L^{\mathfrak{b}}(\Gamma)\right)\right]$, with the understanding that we have fixed the signs on the overcrossings of $p\left(L^{\mathfrak{b}}(\Gamma)\right)$.

Now, given $\Gamma, \Gamma^{\prime} \in \mathcal{A}\left(\Sigma_{g, 1} \times I\right)$, let $L^{\mathfrak{b}}(\Gamma)$ and $L^{\mathfrak{b}}\left(\Gamma^{\prime}\right)$ be the associated $Y$-links in $\Sigma \times[0,1 / 2]$ and $\Sigma \times[1 / 2,1]$ respectively, and let $p_{i}: \Sigma_{g, 1} \times I \rightarrow \Sigma \times\{i\}$ denote the canonical projection. Then we have that

$$
\begin{aligned}
W^{\mathfrak{b}}(\Gamma) \cdot W^{\mathfrak{b}}\left(\Gamma^{\prime}\right) & =\left[\Sigma_{g, 1} \times I, p_{0}\left(l^{\mathfrak{b}}(\Gamma)\right) \cup p_{1 / 2}\left(l^{\mathfrak{b}}\left(\Gamma^{\prime}\right)\right)\right] \\
& =\left[\left(\Sigma_{g, 1} \times I\right)_{C}, p_{0}\left(l^{\mathfrak{b}}(\Gamma)\right) \cup p_{0}\left(l^{\mathfrak{b}}\left(\Gamma^{\prime}\right)\right)\right] \\
& =\left[\left(\Sigma_{g, 1} \times I\right)_{C}, L^{\mathfrak{b}}(\Gamma) \cup L^{\mathfrak{b}}\left(\Gamma^{\prime}\right)\right]
\end{aligned}
$$

where $C$ is a unit-framed trivial link in $\Sigma_{g, 1} \times I$, whose components encircle some crossings of $p_{0}\left(l^{\mathfrak{b}}(\Gamma)\right)$ and $p_{0}\left(l^{\mathfrak{b}}\left(\Gamma^{\prime}\right)\right)$, so that surgery on $C$ brings $p_{0}\left(l^{\mathfrak{b}}(\Gamma)\right)$ below $p_{0}\left(l^{\mathfrak{b}}\left(\Gamma^{\prime}\right)\right)$. The following lemma implies that the result of changing an overcrossing of $L^{\mathfrak{b}}(\Gamma)$ over $L^{\mathfrak{b}}\left(\Gamma^{\prime}\right)$ to an undecrossing can be achieved as a difference of the disjoint union of two graphs minus the disjoint union of two graphs with a leg glued. Together with Equation (6) and the definition of the multiplication $\Gamma * \Gamma^{\prime}$, it implies that

$$
\left[\left(\Sigma_{g, 1} \times I\right)_{C}, L^{\mathfrak{b}}(\Gamma) \cup L^{\mathfrak{b}}\left(\Gamma^{\prime}\right)\right]=W^{\mathfrak{b}}\left(\Gamma * \Gamma^{\prime}\right)
$$

which finishes the proof of the first part of Theorem 5 .

Lemma 3.1. The following identity holds in $\mathcal{G}^{Y} \mathcal{M}\left(\Sigma_{g, 1} \times I\right)$ :

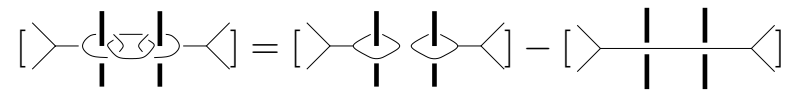

where the framing of the unknot on the left hand side of the equation is +1 and where we alternate with respect to the $Y$-links of the figure. The vertical arcs are arbitrary tubes.

Proof. This follows from the second equality above in $\mathcal{G}^{Y} \mathcal{M}\left(\Sigma_{g, 1} \times I\right)$ and from

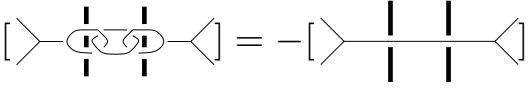

$$
\begin{aligned}
& 2[>d \circlearrowright]=0,
\end{aligned}
$$

see [GGP]. 
The second part of Theorem 5 is obvious from the definition of the Lie bracket.

For the third part, notice that the Lie algebra structure on the quotient $\mathcal{A}^{t}\left(\Sigma_{g, 1} \times I\right) \cong \mathcal{A}^{c}\left(\Sigma_{g, 1} \times\right.$ $I) / \mathcal{A}^{l}\left(\Sigma_{g, 1} \times I\right)$ is given by

$$
\left[\Gamma, \Gamma^{\prime}\right]=\left\langle\Gamma, \Gamma^{\prime}\right\rangle_{1}-\left\langle\Gamma, \Gamma^{\prime}\right\rangle_{1}=-\left[\Gamma, \Gamma^{\prime}\right]^{\omega},
$$

where the last equality follows from the first part of Proposition 2.8 and where $\omega$ is the symplectic form.

Proof. (of Theorem 6 and Remark 2.11) For a surface $\Sigma_{g, 1}$ of genus $g \geq 3$ with one boundary component, Johnson [Jo1] introduced a homomorphism $\tau_{1}: \mathcal{G}_{1} \mathcal{T}_{g, 1} \rightarrow \Lambda^{3}(H)$, where, following the conventions of [Jo1, Chapter 4], $H=H_{1}\left(\Sigma_{g, 1}, \mathbb{Z}\right)$ and $\Lambda^{m}(H)$ is identified with a submodule of the $m$-th tensor power $\mathrm{T}^{k}(H)$ by defining

$$
a_{1} \wedge \cdots \wedge a_{m}=\sum_{\pi \in \operatorname{Sym}_{m}} a_{\pi(1)} \otimes \cdots \otimes a_{\pi(m)} .
$$

In subsequent work, Johnson showed that modulo 2-torsion his homomorphism coincides with the abelianization of $\mathcal{T}_{g, 1}$, thus one gets, over $\mathbb{Q}$, an onto map of Lie algebras $\mathrm{L}\left(\Lambda^{3}(H)\right) \rightarrow \mathcal{G} \mathcal{T}_{g, 1}$.

For the rest of the proof we will work over $\mathbb{Q}$. In [Ha, Section 11] Hain proved that for genus $g \geq 6$, the above map of Lie algebras has kernel generated by quadratic relations $R_{g, 1}$ (Hain's notation for $\mathcal{G}_{n} \mathcal{T}_{g, 1}$ is $\left.\mathfrak{t}_{g, 1}(n)\right)$. Combining the proof of [Ha] Proposition 10.3 with Theorem 11.1 and Proposition 11.4], it follows that the relation set $R_{g, 1}$ is the symplectic submodule of $\mathrm{L}_{2}\left(\Lambda^{3}(H)\right)=\Lambda^{2}\left(\Lambda^{3}(H)\right)$ generated by

$$
\left[x_{1} \wedge x_{2} \wedge y_{2}, x_{3} \wedge x_{4} \wedge y_{4}\right]=0
$$

in terms of a standard symplectic basis $\left\{x_{i}, y_{i}\right\}$ of $H$.

Using the isomorphism $\Lambda^{3}(H) \cong \mathcal{A}_{1}^{t}\left(\Sigma_{g, 1} \times I\right)$ given by mapping $a \wedge b \wedge c \in \Lambda^{3}(H)$ to the degree 1 graph $\Gamma_{a, b, c}$ as in remark 2.11, we obtain a map of Lie algebras $\mathrm{L}\left(\Lambda^{3}(H)\right) \rightarrow \mathcal{A}^{c}\left(\Sigma_{g, 1} \times I\right)$.

Since for every choice of $a \in\left\{x_{1}, x_{2}, y_{2}\right\}$ and $b \in\left\{x_{3}, x_{4}, y_{4}\right\}$ we have $a \cdot b=0$, the first part of Proposition 2.8 implies for every basis $\mathfrak{b}$ we have $\langle a, b\rangle^{\mathfrak{b}}=\langle b, a\rangle^{\mathfrak{b}}$. This implies, by definition, that $\left[\Gamma_{x_{1}, x_{2}, y_{2}}, \Gamma_{x_{3}, x_{4}, y_{4}}\right]=0 \in \mathcal{A}_{2}^{c}\left(\Sigma_{g, 1} \times I\right)$, thus obtaining the desired map $\Phi: \mathcal{G} \mathcal{T}_{g, 1} \cong$ $\mathrm{L}\left(\Lambda^{3}(H)\right) /\left(R_{g, 1}\right) \rightarrow \mathcal{A}^{c}\left(\Sigma_{g, 1} \times I\right)$.

Since $\mathcal{G} \mathcal{T}_{g, 1}$ is generated by its elements of degree 1 , the commutativity of the two diagrams follows by their commutativity in degree 1; the later follows by definition for the first diagram, and by the fact that surgery on a $Y$-link of degree 1 with counterclockwise orientation and leaves decorated by $a, b, c$ is equivalent to cutting, twisting and gluing by an element of the Torelli group (of a surface of genus 3 with one boundary component, imbedded in $\Sigma_{g, 1}$ ) whose image under the Johnson homomorphism is equal to $a \wedge b \wedge c$, see [GGP]. This concludes the proof of Theorem 6 .

We now prove the statements in Remark 2.11. For a closed surface $\Sigma_{g}$ of genus $g \geq 3$, Johnson [Jo1] gave a version of his homomorphism $\tau_{1}: \mathcal{G T}_{g} \rightarrow \Lambda_{0}^{3}(H)$ where $\Lambda_{0}^{3}(H)$ is defined to be the cokernel of the homomorphism $H \rightarrow \Lambda^{3}(H)$ that sends $x$ to $\omega \wedge x$, where $\omega=\sum_{i} x_{i} \wedge y_{i}$ is the symplectic form of $\Sigma_{g}$, for a choice of symplectic basis. Working, from now on, over $\mathbb{Q}$, Johnson [Jo1, Chapter 4] gave an identification of $\Lambda_{0}^{3}(H)$ with $\operatorname{Ker}(C)$, where $C: \Lambda^{3}(H) \rightarrow H$ is given by

$$
C(x \wedge y \wedge z)=2((x \cdot y) z+(y \cdot z) x+(z \cdot x) y)
$$

and - denotes the symplectic form. Explicitly, we will think of $\Lambda_{0}^{3}(H)$ as the submodule of $\Lambda^{3}(H)$ which is generated by elements of the form $2(g-1) a-\omega \wedge C(a)$ for $a \in \Lambda^{3}(H)$. In [Ha, Theorems 1.1 and 10.1] Hain proved that for genus $g \geq 6$, there is an isomorphism of graded Lie algebras $\mathrm{L}\left(\Lambda_{0}^{3}(H)\right) /\left(R_{g}\right) \rightarrow \mathcal{G} \mathcal{T}_{g}$ which, in degree 1 , is the inverse of the Johnson homomorphism, where $R_{g}$ is the symplectic submodule of $\mathrm{L}_{2}\left(\Lambda_{0}^{3}(H)\right)=\Lambda^{2}\left(\Lambda_{0}^{3}(H)\right)$ generated by the relations

$$
\left[2(g-1) x_{1} \wedge x_{2} \wedge y_{2}-x_{1} \wedge \omega, 2(g-1) x_{3} \wedge x_{4} \wedge y_{4}-x_{3} \wedge \omega\right]=0 .
$$


The slight difference of 2 in the relations that Hain gave and the ones mentioned above are due to the difference in the normalization of the $\wedge$-product between Hain and Johnson. The restriction of the map $\mathrm{L}\left(\Lambda^{3}(H)\right) \rightarrow \mathcal{A}^{c}\left(\Sigma_{g, 1} \times I\right) \cong \mathcal{A}^{c}\left(\Sigma_{g} \times I\right) \rightarrow \mathcal{A}^{c}\left(\Sigma_{g} \times I\right) /\left(\Gamma_{\omega}\right)$ to $\mathrm{L}\left(\Lambda_{0}^{3}(H)\right)$ gives a map $\mathrm{L}\left(\Lambda_{0}^{3}(H)\right) \rightarrow \mathcal{A}^{c}\left(\Sigma_{g} \times I\right) /\left(\Gamma_{\omega}\right)$ which sends the relations $R_{g}$ to zero (this really follows from the calculation of the surface with one boundary component together with the fact that $a \wedge \omega \in \Lambda^{3}(H)$ is sent into the ideal $\Gamma_{\omega}$ of $\left.A_{1}^{t}\left(\Sigma_{g} \times I\right)\right)$, thus inducing the desired map $\Phi: \mathcal{G} \mathcal{T}_{g} \rightarrow \mathcal{A}^{c}\left(\Sigma_{g} \times I\right) /\left(\Gamma_{\omega}\right)$. We claim that $W^{\mathfrak{b}}(M): \mathcal{A}\left(\Sigma_{g} \times I\right) \rightarrow \mathcal{G}^{Y} \mathcal{M}\left(\Sigma_{g} \times I\right)$ maps $\Gamma_{\omega}$ to zero. This follows from the identity

$$
\left(\tau_{\partial}\right)^{2 g-2}=\prod_{i}\left[\tau_{x_{i}}, \tau_{y_{i}}\right]
$$

of Dehn twists on the mapping class group of $\Sigma_{g, 1}$ [Mo1, Theorem 5.3], where $x_{i}, y_{i}$ refer to the standard meridian, longitude pairs associated with a symplectic basis of $H_{1}\left(\Sigma_{g, 1}\right)$ and $\partial$ is the boundary curve of $\Sigma_{g, 1}$. Thus we have the relation

$$
1=\prod_{i} \tau_{a}\left[\tau_{x_{i}}, \tau_{y_{i}}\right] \tau_{a^{\prime}}^{-1}
$$

on the mapping class group of $\Sigma_{g}$ (where $a, a^{\prime}$ are simple closed curves in $\Sigma_{g, 1}$ with isotopic images in $\Sigma_{g}$ ), together with the fact surgery along the $Y$-link $\Gamma_{x_{i}, y_{i}, a}$ corresponds to the Dehn twist $\tau_{a}\left[\tau_{x_{i}}, \tau_{y_{i}}\right] \tau_{a^{\prime}}^{-1}$ in $\mathcal{T}_{g},[\mathrm{GGP}]$.

Since $\mathcal{G} \mathcal{T}_{g}$ is generated by its elements of degree 1 , the commutativity of the two diagrams follows by their commutativity in degree 1 ; this is shown in the same way as for a surface with one boundary component. This concludes the proof of Remark 2.11 .

Proof. (of Theorem 7) For a closed surface $\Sigma$ of genus at least 6, Theorem 7 follows from Remark 2.11 and the following Lemma 3.3, perhaps of independent interest. For a closed surface $\Sigma$ of genus less than 6, fix a disk and consider an imbedding of its complement to a surface $\Sigma^{\prime}$ in $M$ of genus at least 6 . Choose a lifting of $\phi$ to a diffeomorphism of the punctured surface that preserves the boundary and extend it trivially to a diffeomorphism of $\phi^{\prime}$ of $\Sigma^{\prime}$. Since the Johnson homomorphism is stable with respect to increase in genus, and since $M_{\phi}=M_{\phi^{\prime}}$, the result follows from the previous case.

Lemma 3.2. If $\Gamma \subseteq M$ is an imbedded graph in $M$ with a distinguished leaf that bounds a surface disjoint from the other leaves of $\Gamma$, then $[M, \Gamma]=0 \in \mathcal{G}^{Y} \mathcal{M}(M)$.

Proof. First of all, recall that $[M, \Gamma]=0$ if any leaf bounds a disk disjoint from the other leaves of $\Gamma$. As explained in [GGP], an alternative way of writing Equation (6) is as follows:

$$
[\mapsto]=[\infty]+[\hookrightarrow]
$$

for arbitrary disjoint imbeddings of two based oriented knots in $M$. Given a based $\operatorname{knot} \alpha$ in $M$, let $\bar{\alpha}$ denote the based knot obtained by a push-off of $\alpha$ in its normal direction (any will do) followed by reversing the orientation.

The above identity implies that $\left[M, \Gamma_{\bar{\alpha}}\right]=-\left[M, \Gamma_{\alpha}\right]$ in $\mathcal{G}^{Y} \mathcal{M}(M)$, where $\Gamma_{\kappa}$ is any imbedded graph in $M$ with a distinguished leaf the based oriented knot $\kappa$ in $M$.

Given $\Gamma$ as in the statement of the lemma, it follows that its distinguished leaf is the connected sum of disjoint based knots of the form $\alpha_{i} \sharp \beta_{i} \sharp \overline{\alpha_{i}} \sharp \overline{\beta_{i}}$; thus it follows from the above discussion that $[M, \Gamma]=0$ in $\mathcal{G}^{Y} \mathcal{M}(M)$.

Lemma 3.3. Given an imbedding $\iota: N \rightarrow M$ of (not-necessarily closed) 3-manifolds, and links $\mathfrak{b}(N)$ (resp. $\mathfrak{b}(M))$ in $N$ (resp. $M)$ representing a basis of $H_{1}(N)$ (resp. $H_{1}(M)$ ), there is an 
induced map $\iota_{*}: \mathcal{A}(N) \rightarrow \mathcal{A}(M)$ induced by $\iota_{*}: H_{1}(N) \rightarrow H_{1}(M)$ on the colorings of the legs of the graphs and a diagram

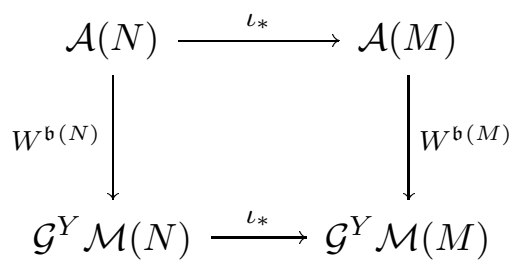

that commutes up to $W^{\mathfrak{b}(M)}\left(\mathcal{A}^{l}(M)\right)$. In particular, for $\Gamma \in \mathcal{A}^{t}(N)$ we have

$$
\iota_{*} W^{\mathfrak{b}(N)}(\Gamma)=W^{\mathfrak{b}(M)}\left(\iota_{*}(\Gamma)\right)+\text { loops. }
$$

Proof. Fix a graph $\Gamma \in \mathcal{A}^{t}(N)$. Let $\Gamma_{L}$ (resp. $\Gamma_{L^{\prime}}$ ) denote the $Y$-links in $M$ (with leaves $L$ (resp. $\left.L^{\prime}\right)$ ) such that

$$
\iota_{*} W^{\mathfrak{b}(N)}(\Gamma)=\left[M, \Gamma_{L}\right] \text { and } W^{\mathfrak{b}(M)}\left(\iota_{*} \Gamma\right)=\left[M, \Gamma_{L^{\prime}}\right] .
$$

It follows by definition that $L$ and $L^{\prime}$ are homologous links in $M$. After choosing a common base point for each pair $\left(L_{i}, L_{i}^{\prime}\right)$ of components of $L$ and $L^{\prime}$, it follows that the connected sum $L_{i} \sharp \overline{L_{i}^{\prime}}$ is nullhomologous in $M$ and thus bounds a surface $\Sigma_{i}$ in $M$. The surface $\Sigma_{i}$ might intersect the other components of $L$ or $L^{\prime}$ at finitely many points; however by deleting disks around the points of intersection of $\Sigma_{i}$ with $L \cup L^{\prime}$, we can find a nullhomotopic based link $L_{i}^{\prime \prime}$ and a surface $\Sigma_{i}^{\prime}$ disjoint from $L \cup L^{\prime}$ with based boundary such that $L_{i} \sharp \overline{L_{i}^{\prime}}=L_{i}^{\prime \prime} \sharp \partial \Sigma_{i}^{\prime}$. Equation (8) and Lemma 3.2 imply that $\left[M, \Gamma_{L}\right]-\left[M, \Gamma_{L^{\prime}}\right]$ is a sum of terms over $Y$-links $\Gamma_{\kappa}$ in $M$ which are trees, with at least one component $\kappa$ being nullhomotopic. By choosing a sequence of crossing changes (represented by a unit-framed trivial link $C(\kappa)$ ) that trivialize $\kappa$ and using Lemma 3.1, it follows that $\left[M, \Gamma_{\kappa}\right]=$ $\left[M, \Gamma_{\text {trivial }}\right]=0$ modulo terms that involve joining some legs of $\Gamma$ (thus modulo terms that involve graphs with loops), which concludes the proof.

\section{Massey PROduCts AND The Johnson homomorPhism}

4.1. Universal Massey products. In this section, homology will be with integer coefficients, unless otherwise stated. A useful tool in the proof of Theorem 1 is a five-term exact sequence of Stallings [St]: given a short exact sequence of groups $1 \rightarrow H \rightarrow G \rightarrow K \rightarrow 1$, there is an associated five-term exact sequence

$$
H_{2}(G) \rightarrow H_{2}(K) \rightarrow H /[G, H] \rightarrow H_{1}(G) \rightarrow H_{1}(K) \rightarrow 1 .
$$

Applying the five-term sequence to the exact sequence $1 \rightarrow R \rightarrow F \rightarrow G \rightarrow 1$, (where $F$ is a free group) we get Hopf's theorem [Ho]

$$
H_{2}(G) \cong(R \cap[F, F]) /[R, F] \text {, and in particular, } H_{2}\left(F / F_{n}\right) \cong F_{n} / F_{n+1} .
$$

In the rest of this section, we will give a proof of Theorem 1 and its corollaries. We will follow a rather traditional notation involving local coordinates, [Ma, FS, Dw]. Let $F$ denote the free group with basis $\left(x_{1}, \ldots, x_{m}\right)$; we will denote by the same name the corresponding basis of $H=H_{1}(F)$. Let $\left(u_{1}, \ldots, u_{m}\right)$ be the dual basis of $H^{1}(F) \cong H^{1}\left(F / F_{n}\right)$. The graded vector space $\bigoplus_{n} F_{n} / F_{n+1}$ has the structure of a Lie algebra induced by commutator, and is naturally identified with the free Lie algebra $\mathrm{L}(H)=\bigoplus_{n} \mathrm{~L}_{n}(H)$.

We consider the Magnus expansion, [MKS]. Let $\mathbb{Z} \llbracket t_{1}, \ldots, t_{m} \rrbracket$ denote the power series ring in non-commuting variable $\left\{t_{1}, \ldots, t_{m}\right\}$. Define $\delta: F \rightarrow \mathbb{Z} \llbracket t_{1}, \ldots, t_{m} \rrbracket$ to be the multiplicative map defined by $\delta\left(x_{i}\right)=1+t_{i}$. This is an imbedding and induces imbeddings $\delta_{n}: \mathrm{L}_{n}(H)=F_{n} / F_{n+1} \rightarrow$ $\mathbb{Z} \llbracket t_{1}, \ldots, t_{m} \rrbracket_{n}$, where $\mathbb{Z} \llbracket t_{1}, \ldots, t_{m} \rrbracket_{n}$ is the subspace of homogeneous polynomials of degree $n$.

We also recall the isomorphism $H_{2}\left(F / F_{n}\right) \cong F_{n} / F_{n+1}=\mathrm{L}_{n}(H)$ from above. We now describe the Massey product structure on $H^{1}\left(F / F_{n}\right)$. For a sequence $I=\left(i_{1}, \ldots, i_{r}\right)$ of numbers $i_{j} \in\{1, \ldots, m\}$ 
(of length $|I| \stackrel{\text { def }}{=} r$ ), we will let $u_{I}$ denote the sequence $\left(u_{i_{1}}, \ldots, u_{i_{r}}\right)$ and, if each $u_{i} \in H^{1}\left(F / F_{n}\right)$ we let $\left\langle u_{I}\right\rangle$ denote the length $r$ Massey product; we also let $t_{I}$ denote the element $\prod_{j=1}^{r} t_{i_{j}}$.

Proposition 4.1. Any Massey product $\left\langle u_{I}\right\rangle$ of $F / F_{n}$ vanishes if $|I|<n$. The action of any Massey product $\left\langle u_{I}\right\rangle$ on $H_{2}\left(F / F_{n}\right) \cong F_{n} / F_{n+1}=\mathrm{L}_{n}(H) \subseteq \mathbb{Z} \llbracket t_{1}, \ldots, t_{m} \rrbracket$ is determined by the formula

$$
\left\langle u_{I}\right\rangle \cdot t_{J}= \begin{cases}1 & \text { if } I=J \text { and }|I|=n \\ 0 & \text { otherwise }\end{cases}
$$

In other words, the set $\left\{\left\langle u_{I}\right\rangle|| I \mid=n\right\}$ defines the basis of $\mathbb{Z} \llbracket t_{1}, \ldots, t_{m} \rrbracket_{n}^{*}$ dual to $\left\{t_{I}|| I \mid=n\right\}$.

See [O2] for a slightly less explicit version of this theorem.

Proof. This follows easily from [FS]. Suppose $w \in F_{n}$ is some $n$-fold commutator. Then consider the one-relator group $G=F /\langle w\rangle$ and the projection $p: F / F_{n} \rightarrow G$. Consider $I$ of length $r \leq n$. By induction we can assume that $\left\langle u_{I}\right\rangle$ is uniquely defined in $F / F_{n}$ and by [FS], it is well-defined in $G$. Moreover, by naturality under $p^{*}$, they take the same value on $w$. If $r<n$ this is zero by [FS]. Since this holds for all $w$ it follows that $\left\langle u_{I}\right\rangle=0$. If $r=n$ Equation (9) follows directly from the formula in [FS].

Corollary 4.2. Let $p: F \rightarrow \pi$ be a 2-equivalence. Then $p$ is an n-equivalence if and only if all Massey products in $H_{1}(\pi)$ of length less than $n$ vanish.

See also [CGO, Proposition 6.8].

Proof. The "if" part follows directly from the above proposition. To prove the "only if" part we proceed by induction on $n$. The inductive step presents us with a map $\pi \rightarrow \pi / \pi_{n-1} \cong F / F_{n-1}$; consider the diagram

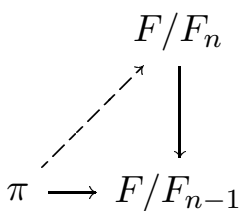

The obstruction to lifting this map is the pullback of the characteristic class in $H^{2}\left(F / F_{n-1} ; F_{n-1} / F_{n}\right)$ of the central extension $F_{n-1} / F_{n} \rightarrow F / F_{n-1} \rightarrow F / F_{n}$. But Proposition 4.1 implies that $H^{2}\left(F / F_{n-1}\right)$ is generated by Massey products of length $n-1$ and so the pullback is zero if and only if all Massey products of length $n-1$ vanish in $H^{2}(\pi)$. Thus, we can inductively lift the map to $\pi \rightarrow F / F_{n}$, thus to a map $\pi / \pi_{n} \rightarrow F / F_{n}$, which is still a 2-equivalence.

On the other hand, since $p$ is a 2-equivalence, it induces an onto map $F / F_{n} \rightarrow \pi / \pi_{n}$, which is also a 2-equivalence. Composing with the map $\pi / \pi_{n} \rightarrow F / F_{n}$, we get an endomorphism of $F / F_{n}$ which is a 2-equivalence. Stalling's theorem [St] implies that this endomorphism of $F / F_{n}$ is an isomorphism which implies that the map $F / F_{n} \rightarrow \pi / \pi_{n}$ is one-to-one and thus an $n$-equivalence.

This proves the first assertion of Theorem 1.

Corollary 4.3. Suppose $p: F \rightarrow \pi$ is an n-equivalence. Then there is an exact sequence:

$$
H_{2}(\pi) \stackrel{\hat{p}}{\rightarrow} F_{n} / F_{n+1} \stackrel{p_{*}}{\rightarrow} \pi_{n} / \pi_{n+1} \rightarrow 0,
$$

where $\hat{p}$ is defined by the formula

$$
\hat{p}(\alpha)=\sum_{I}\left(\left\langle u_{I}\right\rangle \cdot \alpha\right) t_{I},
$$

where $\alpha \in H_{2}(\pi)$, the summation is over I of length $n, \cdot: H^{*} \otimes H_{*} \rightarrow \mathbb{Z}$ is the evaluation map, and where the right hand side is asserted to lie in $\mathrm{L}_{n}(H)=F_{n} / F_{n+1} \subseteq \mathbb{Z} \llbracket t_{1}, \ldots, t_{m} \rrbracket$. 
Proof. Apply Stallings five-term exact sequence to the short exact sequence of groups $1 \rightarrow \pi_{n} \rightarrow$ $\pi \rightarrow \pi / \pi_{n} \rightarrow 1$ to obtain

$$
H_{2}(\pi) \rightarrow H_{2}\left(\pi / \pi_{n}\right) \rightarrow \pi_{n} / \pi_{n+1} \rightarrow 1 .
$$

Combining this with the map $p$ gives the commutative diagram

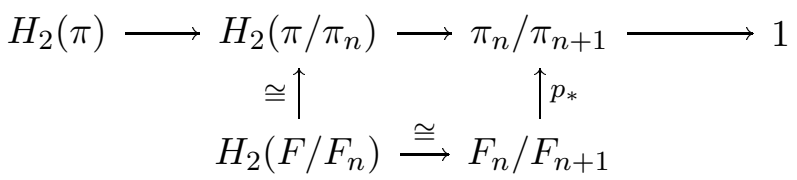

This diagram yields the exact sequence of the corollary, where $\hat{p}$ is defined as the composition

$$
H_{2}(\pi) \rightarrow H_{2}\left(\pi / \pi_{n}\right) \cong H_{2}\left(F / F_{n}\right) \cong F_{n} / F_{n+1}
$$

To prove the formula for $\hat{p}$ first note that, for any $\alpha \in H_{2}\left(F / F_{n}\right) \cong F_{n} / F_{n+1}=\mathrm{L}_{n}(H) \subseteq$ $\mathbb{Z} \llbracket t_{1}, \ldots, t_{m} \rrbracket_{n}$ we have

$$
\alpha=\sum_{I}\left(\left\langle u_{I}\right\rangle \cdot \alpha\right) t_{I}
$$

as follows directly from Proposition 4.1. But now the corollary follows from the definition of $\hat{p}$ and naturality.

This proves the second assertion of Theorem 1 for $K(\pi, 1)$ spaces.

Remark 4.4. Given two choices $p, p^{\prime}$ of maps as in Corollary 4.3, we get a commutative diagram:

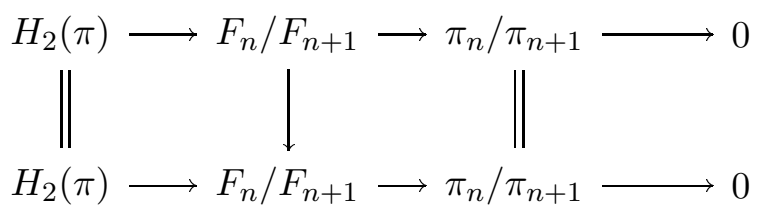

where the middle map is the automorphism of $F_{n} / F_{n+1} \cong H_{2}\left(F / F_{n}\right)$ defined by the composition of isomorphisms

$$
H_{2}\left(F / F_{n}\right) \stackrel{p_{*}}{\rightarrow} H_{2}\left(\pi / \pi_{n}\right) \stackrel{p_{*}^{\prime}}{\longleftarrow} H_{2}\left(F / F_{n}\right) .
$$

In particular, if $p^{\prime} \equiv p \bmod F_{2}$ then the two exact rows are identical.

The third assertion of Theorem 1 for $K(\pi, 1)$ spaces follows from the following

Proposition 4.5. Let $p: F \rightarrow \pi$ be an n-equivalence and $\alpha_{1}, \ldots, \alpha_{n+1} \in H^{1}(\pi)$. Then we have:

$$
\alpha_{1} \smile\left\langle\alpha_{2}, \ldots, \alpha_{n+1}\right\rangle=\left\langle\alpha_{1}, \ldots, a_{n}\right\rangle \smile \alpha_{n+1} .
$$

See also $[\mathrm{Kr}]$ for a related result.

Proof. We will use Dwyer's formulation [Dw] of the Massey products. Choose cocycles $a_{i}$ representing $\alpha_{i}$, for $1 \leq i \leq n+1$. Since we are assuming all Massey products of length less than $n$ are defined and vanish, we can choose cochains $a_{i j}$, for $1 \leq i<j \leq n+2$, with the exception of the three cases

$$
i=1, j=n+1 \quad i=2, j=n+2 \quad i=1, j=n+2
$$

so that $a_{i, i+1}=a_{i}$ and $\delta a_{r s}=\sum_{r<i<s} a_{r i} \smile a_{i s}$. For two of the three exceptional cases the cochains

$$
b_{1, n+1}=\sum_{1<i<n+1} a_{1 i} \smile a_{i, n+1} \quad \text { and } \quad b_{2, n+2}=\sum_{2<i<n+2} a_{2 i} \smile a_{i, n+2}
$$

the $b_{i j}$ are cocycles but not necessarily coboundaries. In fact they represent the Massey products $\left\langle\alpha_{1}, \ldots, \alpha_{n}\right\rangle$ and $\left\langle\alpha_{2}, \ldots, \alpha_{n+1}\right\rangle$ respectively.

Thus $\left\langle\alpha_{1}, \ldots, \alpha_{n}\right\rangle \smile \alpha_{n+1}$ is represented by the cocycle $b_{1, n+1} \smile a_{n+1, n+2}$ and $\alpha_{1} \smile\left\langle\alpha_{2}, \ldots, \alpha_{n+1}\right\rangle$ is represented by the cocycle $a_{12} \smile b_{2, n+2}$. 
Now consider the cochain

$$
c=\sum_{1<i<r<n+2} a_{1 i} \smile a_{i r} \smile a_{r, n+2}
$$

By grouping the terms in one way we see that

$$
\begin{aligned}
c & =a_{12} \smile\left(\sum_{2<r<n+2} a_{2 r} \smile a_{r, n+2}\right)+\sum_{2<i<r<n+2}\left(a_{1 i} \smile a_{i r} \smile a_{r, n+2}\right) \\
& =a_{12} \smile b_{2, n+2}+\sum_{2<i<r<n+2}\left(a_{1 i} \smile \delta a_{i, n+2}\right)
\end{aligned}
$$

Grouping the terms in another way gives

$$
\begin{aligned}
c & =\sum_{1<i<r<n+1}\left(a_{1 i} \smile a_{i r} \smile a_{r, n+2}\right)+\left(\sum_{1<i<n+1} a_{1 i} \smile a_{i, n+1}\right) \smile a_{n+1, n+2} \\
& =\sum_{1<r<n+1}\left(\delta a_{1 r} \smile a_{r, n+2}\right)+b_{1, n+1} \smile a_{n+1, n+2}
\end{aligned}
$$

Now subtracting these two formulae for $c$ gives

$$
\begin{aligned}
a_{12} \smile b_{2, n+2}-b_{1, n+1} \smile a_{n+1, n+2} & =\sum_{1<r<n+1} \delta a_{1 r} \smile a_{r, n+2}-\sum_{2<i<n+2} a_{1 i} \smile \delta a_{i, n+2} \\
& =\delta\left(\sum_{2<i<n+1} a_{1 i} \smile a_{i, n+2}\right)
\end{aligned}
$$

since $a_{12}$ and $a_{n+1, n+2}$ are cocycles. Since the left side of this equation represents

$$
\alpha_{1} \smile\left\langle\alpha_{2}, \ldots, \alpha_{n+1}\right\rangle-\left\langle\alpha_{1}, \ldots, \alpha_{n}\right\rangle \smile \alpha_{n+1}
$$

and the right side is a coboundary the proof is complete.

This concludes the proof of Theorem 1 for $K(\pi, 1)$ spaces. The general case follows from the fact that the canonical map $X \rightarrow K(\pi, 1)$ induces an onto map $H_{2}(X) \rightarrow H_{2}(\pi)$.

Proof. (of Corollary 2.2) The first part is immediate from Theorem 1, using the Poincaré duality isomorphism $H^{*}=H_{1}(M, \mathbb{Q})=H^{2}(M, \mathbb{Q})$. In local coordinates, it implies (see Corollary 4.3) that the Massey product

$$
\mu_{n}(M) \in H \otimes \mathrm{L}_{n}(H) \cong \operatorname{Hom}\left(H^{1}(M), \mathrm{L}_{n}(M)\right)
$$

is given by

$$
\mu_{n}(M)(u)=\sum_{I}[M] \frown\left(u \smile\left\langle u_{I}\right\rangle\right) t_{I},
$$

where $u \in H^{1}(M)$, the summation is over $I$ of length $n, \smile$ indicates cup product and $[M] \frown$ indicates cap product with the fundamental homology class of $M$. Let $[\cdot]: H \otimes \mathrm{L}(H) \rightarrow \mathrm{L}(H)$ be the Lie algebra bracket, defined by $[a \otimes b]=[a, b]$, for $a \in H, b \in \mathrm{L}(H)$. If we regard $\mathrm{L}(H) \subseteq \mathbb{Z} \llbracket t_{1}, \ldots, t_{m} \rrbracket$ then $[\cdot]$ can be expressed by the formula

$$
\left[x_{i} \otimes c\right]=\left(1+t_{i}\right) c-c\left(1+t_{i}\right)=t_{i} c-c t_{i} .
$$

Equation (10) implies that

$$
\mu_{n}(M)=\sum_{i, I} x_{i} \otimes\left([M] \frown\left(u_{i} \smile\left\langle u_{I}\right\rangle\right)\right) t_{I}
$$

and so

$$
\left[\mu_{n}(M)\right]=\sum_{i, I}[M] \frown\left(u_{i} \smile\left\langle u_{I}\right\rangle\right)\left(t_{i} t_{I}-t_{I} t_{i}\right) .
$$


Thus Corollary 2.2 follows from the third assertion of Theorem 1 (or its coordinate version, Proposition 4.5).

\subsection{Realization results.}

Proof. (of Theorem 3) Given an element $\phi \in \mathrm{A}_{0}\left(F / F_{n}\right)$ we construct maps $f^{ \pm}: \Sigma_{g, 1} \rightarrow K\left(F / F_{n}, 1\right)$, where $f_{*}^{+}: \pi_{1}\left(\Sigma_{g, 1}\right) \rightarrow F / F_{n}$ corresponds to the canonical projection $p: F \rightarrow F / F_{n}$ under the identification of $\pi_{1}\left(\Sigma_{g, 1}\right)$ with $F$, and $f_{*}^{-}=\phi \circ p$. Since $\phi_{*}\left(\omega_{g}\right)=\omega_{g}$, we have $f^{+}\left|\partial \Sigma_{g, 1} \simeq f^{-}\right| \partial \Sigma_{g, 1}$ and so we can combine the two maps to define a map $f: \hat{\Sigma}_{g, 1} \rightarrow K\left(F / F_{n}, 1\right)$, where $\hat{\Sigma}_{g, 1}$ is the double of $\Sigma_{g, 1}$. We would like to extend $f$ to a map $\Phi: M \rightarrow K\left(F / F_{n}, 1\right)$, for some compact orientable 3-manifold with $\partial M=\hat{\Sigma}_{g, 1}$ the obstruction to the existence of $\Phi$ is the element $\theta \in$ $\Omega_{2}\left(F / F_{n}\right) \cong H_{2}\left(F / F_{n}\right)$ represented by $f$, where $\Omega_{*}\left(F / F_{n}\right)$ are the oriented bordism groups of $F / F_{n}$. Since $H_{2}\left(F / F_{n}\right) \neq 0$ we must be careful in our choices to assure that $\theta=0$. Redo the construction of $f^{+}, f^{-}$and $f$ but using $K\left(F / F_{n+1}, 1\right)$ instead of $K\left(F / F_{n}, 1\right)$ and using a lift of $\phi$ to an automorphism $\bar{\phi}$ of $F / F_{n+1}$ instead of $\phi$. Our restriction on $\phi$ assures that $\bar{\phi}\left(\omega_{g}\right)=\omega_{g}$ and so we obtain $\bar{f}: \hat{\Sigma}_{g, 1} \rightarrow K\left(F / F_{n+1}, 1\right)$ and an obstruction element $\bar{\theta} \in \Omega_{2}\left(F / F_{n+1}\right)$. Now this element may not be zero, but since the projection map $H_{2}\left(F / F_{n+1}\right) \rightarrow H_{2}\left(F / F_{n}\right)$ is zero, and clearly $\bar{\theta}$ maps to $\theta$, we conclude that $\theta=0$. Thus $f$ extends to the desired $\Phi: M \rightarrow K\left(F / F_{n}, 1\right)$.

Let $i^{ \pm}: \Sigma_{g, 1} \rightarrow \partial M$ be the obvious diffeomorphisms onto the domains of $f^{ \pm}$. It is clear that if $M$ were a homology cylinder over $\Sigma^{+}$, then $\sigma_{n}(M)=\phi$. But this is not necessarily true and so we will perform surgery on the map $\Phi$, adapting the arguments in $[\mathrm{KM}]$ to our situation. See also [Tu, Theorem 1] for similar surgery arguments which are used to show that any finite 3-dimensional Poincaré complex is homology equivalent to a closed 3-manifold.

Lemma 4.6. Suppose $\alpha \in \operatorname{Ker} \Phi_{*}: H_{1}(M) \rightarrow H$. Then there exists $\bar{\alpha} \in \pi_{1}(M)$ such that $\bar{\alpha} \in \operatorname{Ker} \Phi_{*}: \pi_{1}(M) \rightarrow F / F_{n}$ and $\bar{\alpha}$ represents $\alpha$.

Proof. If $\bar{\alpha} \in \pi_{1}(M)$ is any representative of $\alpha$, then $\Phi_{*}(\bar{\alpha}) \in F_{2} / F_{n}$. Choose an element $\beta \in$ $\pi_{1}\left(\Sigma^{+}\right)_{2}$ so that $\Phi_{*}(\beta)=f_{*}^{+}(\beta)=\bar{\alpha}$. Then $\bar{\alpha} \beta^{-1} \in \operatorname{Ker} \Phi_{*}$ and $\bar{\alpha} \beta^{-1}$ represents $\alpha$.

Thus for any $\alpha \in \operatorname{Ker} \Phi_{*}: H_{1}(M) \rightarrow H$ we can do surgery on a curve representing $\alpha$ and extend $F$ over the trace of the surgery.

The first step in killing $\operatorname{Ker} \Phi_{*}$ will be to kill the torsion-free part. Note that $H_{1}(M) \cong$ $H_{1}\left(\Sigma^{+}\right) \oplus \operatorname{Ker} \Phi_{*}$, since $\Phi_{*} \circ i_{*}^{+}$is an isomorphism, and so, under the canonical map $H_{1}(M) \rightarrow$ $H_{1}(M, \partial M)$, Ker $\Phi_{*}$ maps onto $H_{1}(M, \partial M)$. Choose an element $\alpha \in \operatorname{Ker} \Phi_{*}$ which maps to a primitive element of $H_{1}(M, \partial M)$. Now surgery on a simple closed curve $C$ representing $\alpha$ will produce a new manifold $M^{\prime}$ so that, if $\beta \in H_{1}\left(M^{\prime}\right)$ is the element represented by the meridian of $C$, then

$$
H_{1}(M) /\langle\alpha\rangle \cong H_{1}\left(M^{\prime}\right) /\langle\beta\rangle
$$

(see $[\mathrm{KM}]$ ). Since $\alpha$ is primitive in $H_{1}(M, \partial M)$, there is a 2-cycle $z$ in $M$ whose intersection number with $C$ is +1 . Thus the intersection of $z$ with $M^{\prime}$ is a 2 -chain whose boundary is $\beta$. So, by Equation (11), $H_{1}\left(M^{\prime}\right) \cong H_{1}(M) /\langle\alpha\rangle$.

A sequence of such surgeries will kill the torsion-free part of $H_{1}(M, \partial M)$. But this implies that $\operatorname{Ker} \Phi_{*}$ is torsion by the following simple homology argument. Consider the exact sequence:

$$
0 \rightarrow H_{2}(M) \rightarrow H_{2}(M, \partial M) \rightarrow H_{1}(\partial M) \rightarrow H_{1}(M) \rightarrow H_{1}(M, \partial M) \rightarrow 0
$$

Since $\operatorname{rank} H_{2}(M)=\operatorname{rank} H_{1}(M, \partial M)=0$ and $H_{1}\left(\Sigma^{+}\right)$imbeds into $H_{1}(M), \operatorname{rank} H_{2}(M, \partial M)=$ $\operatorname{rank} H_{1}(M) \geq 2 g$. But, since $\operatorname{rank} H_{1}(\partial M)=4 g$, we conclude that rank $H_{1}(M)=2 g$. Therefore

$$
2 g=\operatorname{rank} H_{1}(M)=\operatorname{rank} H_{1}\left(\Sigma^{+}\right)+\operatorname{rank} \operatorname{Ker} \Phi_{*}
$$

and so $\operatorname{rank} \operatorname{Ker} \Phi_{*}=0$. 
We now follow the argument in $[\mathrm{KM}]$ to kill the torsion group $T=\operatorname{Ker} \Phi_{*}$. The linking pairing $l: T \otimes T \rightarrow \mathbb{Q} / \mathbb{Z}$ is non-singular since $T=$ tors $H_{1}(M)$ maps isomorphically to tors $H_{1}(M, \partial M)=$ $H_{1}(M, \partial M)$. According to [KM, Lemma 6.3] if, for $\alpha \in T, l(\alpha, \alpha) \neq 0$, then we can choose the normal framing to any closed curve $C$ representing $\alpha$ so that the element $\beta \in H_{1}\left(M^{\prime}\right)$ is of finite order smaller than the order of $\alpha$. Thus the torsion subgroup of $H_{1}\left(M^{\prime}\right)$ is smaller than $T$. Continuing in this way we reach the point where all the self-linking numbers are 0 . According to [KM, Lemma 6.5] this implies that $T$ is a direct sum of copies of $\mathbb{Z} / 2$. Now choose any non-zero element $\alpha \in T$. We will show that surgery on $\alpha$ reduces the rank of $H_{1}(M, \partial M ; \mathbb{Z} / 2)$. Denote by $V$ the trace of the surgery and $M^{\prime}$ the result of the surgery. Then we have a diagram of homology groups (coefficients in $\mathbb{Z} / 2$ ) with exact row:

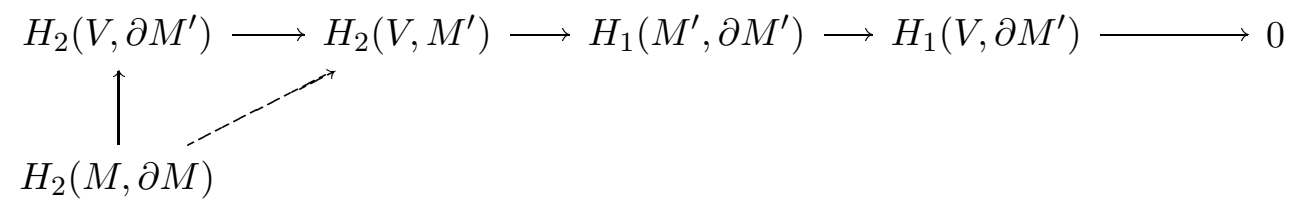

Now $H_{1}\left(V, \partial M^{\prime}\right) \cong H_{1}(M, \partial M) /\langle\alpha\rangle$ and so has rank one less than $H_{1}(M, \partial M)$. Since $H_{2}\left(V, M^{\prime}\right)$ is generated by the transverse disk bounded by the meridian curve representing $\beta$, the dotted arrow can be interpreted as the $(\mathbb{Z} / 2)$ intersection number with $\alpha$. By Poincaré duality this map is nonzero and so $H_{1}\left(M^{\prime}, \partial M^{\prime}\right) \cong H_{1}\left(V, \partial M^{\prime}\right)$, proving the claim. As in $[\mathrm{KM}]$ we can assume the normal framing chosen so that $\beta$ has order 2 or $\infty$. Thus the possibilities for $H_{1}\left(M^{\prime}, \partial M^{\prime} ; \mathbb{Z}\right)$ are either $\mathbb{Z} \oplus(s-2) \mathbb{Z} / 2$ or $\mathbb{Z} / 4 \oplus(s-2) \mathbb{Z} / 2$, where $s=\operatorname{rank} H_{1}(M, \partial M)$. We can then do a surgery to kill the $\mathbb{Z}$ factor, in the first case, or reduce the order of $H_{1}\left(M^{\prime}, \partial M^{\prime} ; \mathbb{Z}\right)$, in the second case. Continuing this way we eventually kill $\operatorname{Ker} \Phi_{*}$, producing the desired $(M, \Phi)$.

Proof. (of Proposition 2.5) Let $\mathrm{D}_{n}^{\mathrm{a}}(H)$ denote the kernel of the natural projection $\mathrm{A}_{0}\left(F / F_{n+1}\right) \rightarrow$ $\mathrm{A}_{0}\left(F / F_{n}\right)$. We first construct a map $D_{n}: \mathrm{D}_{n}^{\mathrm{a}}(H) \rightarrow \mathrm{D}_{n}(H)$ as follows. If $h \in \mathrm{D}_{n}^{\mathrm{a}}(H)$ we can write $h(a)=a \psi(a)$, where $\psi(a) \in F_{n} / F_{n+1} \cong \mathrm{L}_{n}(H)$. Then, we define $D_{n}(h)([a])=\psi(a)$, where $[a] \in H$ and $a \in F / F_{n}$ is a lift of $[a]$. Using the isomorphism $\operatorname{Hom}\left(H, \mathrm{~L}_{n}(H)\right) \cong H \otimes \mathrm{L}_{n}(H)$ this defines a map (denoted by the same name)

$$
\mathrm{D}_{n}^{\mathrm{a}}(H) \rightarrow H \otimes \mathrm{L}_{n}(H)
$$

with corresponding description in local coordinates given by

$$
D_{n}(h)=\sum_{i} x_{i} \otimes \psi\left(y_{i}\right)-y_{i} \otimes \psi\left(x_{i}\right) \in H \otimes \mathrm{L}_{n}(H) .
$$

If $h \in \mathrm{D}_{n}^{\mathrm{a}}(H)$, as above, then $\prod_{i}\left[h\left(x_{i}\right), h\left(y_{i}\right)\right] \equiv \prod_{i}\left[x_{i}, y_{i}\right] \bmod F_{n+2}$ and so

$$
\prod_{i}\left[x_{i} \psi\left(x_{i}\right), y_{i} \psi\left(y_{i}\right)\right] \equiv \prod_{i}\left[x_{i}, y_{i}\right]\left[\psi\left(x_{i}\right), y_{i}\right]\left[x_{i}, \psi\left(y_{i}\right)\right] \bmod F_{n+2}
$$

Therefore $\prod_{i}\left[\psi\left(x_{i}\right), y_{i}\right]\left[x_{i}, \psi\left(y_{i}\right)\right] \in F_{n+2}$, which implies that $D_{n}(h) \in \mathrm{D}_{n}(H)$.

It is clear that $D_{n}$ is one-to-one. We now show that it is onto. Suppose we have an element $\theta=\sum_{i}\left(x_{i} \otimes \alpha_{i}-y_{i} \otimes \beta_{i}\right) \in \mathrm{D}_{n}(H)$. Lift $\alpha_{i}, \beta_{i}$ into $F_{n}$ (denoted by the same symbols) and define an endomorphism $h$ of $F$ by

$$
h\left(x_{i}\right)=x_{i} \alpha_{i}, h\left(y_{i}\right)=y_{i} \beta_{i} .
$$

It follows by Stalling's theorem [St] that $h$ induces an automorphism of $F / F_{n+1}$ which restricts to the identity automorphism of $F / F_{n}$. We note that

$$
h\left(\prod_{i}\left[x_{i}, y_{i}\right]\right)=\prod_{i}\left[x_{i} \alpha_{i}, y_{i} \beta_{i}\right] \equiv \prod_{i}\left[x_{i}, y_{i}\right]\left[x_{i}, \alpha_{i}\right]\left[\beta_{i}, y_{i}\right] \bmod F_{n+2}
$$


But $\prod_{i}\left[x_{i}, \alpha_{i}\right]\left[\beta_{i}, y_{i}\right]$ represents the image of theta under the Lie bracket $H \otimes \mathrm{L}_{n}(H) \rightarrow \mathrm{L}_{n+1}(H)$, which vanishes since $\theta \in \mathrm{D}_{n}(H)$; thus $\prod_{i}\left[x_{i}, \alpha_{i}\right]\left[\beta_{i}, y_{i}\right] \in F_{n+2}$. This shows that $h \in \mathrm{A}_{0}\left(F / F_{n+1}\right)$ and clearly $D_{n}(h)=\theta$.

The fact that the projection $\mathrm{A}_{0}\left(F / F_{n+1}\right) \rightarrow \mathrm{A}_{0}\left(F / F_{n}\right)$ is onto follows immediately from Theorem 3 . It is not hard, however, to give a direct argument; we leave this as an exercise for the reader. Finally, it is clear by the definitions that the diagram in Proposition 2.5 commutes, and that the sequence below it is exact.

Remark 4.7. The action of $\mathrm{A}_{0}\left(F / F_{n}\right)$ on $\mathrm{D}_{n}^{\mathrm{a}}(H) \cong \mathrm{D}_{n}(H)$ induced by conjugation by elements of $\mathrm{A}_{0}\left(F / F_{n+1}\right)$ coincides with the natural action of $\mathrm{A}_{0}\left(F / F_{2}\right) \cong \operatorname{Sp}(2 g, \mathbb{Z})$ on $\mathrm{D}_{n}(H)$, via the projection $\mathrm{A}_{0}\left(F / F_{n}\right) \rightarrow \mathrm{A}_{0}\left(F / F_{2}\right)$.

Proof. (of Theorem 4) Let $M \in \mathcal{H}_{g, 1}$ and define $S(M)^{\circ}=T_{+} \cup M \cup T_{-}$, where $T_{ \pm}$are two copies of the solid handlebody $T$ of genus $g$, which are attached to $\partial M$ via the diffeomorphisms $i^{ \pm}$so that, referring to a basis $\left\{x_{i}, y_{i}\right\}$ of $F$ corresponding to a symplectic basis of $H$, the $\left\{x_{i}\right\}$ are represented by the boundaries of meridian disks in $T$. Thus $\pi_{1}(T)=F^{\prime}$, the free group generated by $\left\{y_{i}\right\}$ (or, more precisely, their images in $\left.\pi_{1}(T)\right) . S(M)^{o}$ is a 3-manifold with boundary $S^{2}$, which we can fill-in to obtain a closed 3-manifold $S(M)$. If $M \in \mathcal{H}_{g, 1}[n]$, then the inclusion $T_{+} \subseteq S(M)$ induces an isomorphism $p: F^{\prime} / F_{n}^{\prime} \cong \pi_{1}(S(M)) / \pi_{1}(S(M))_{n}$ and we can consider $\mu_{n}(S(M), p) \in \mathrm{D}_{n}\left(H^{\prime}\right)$, where $H^{\prime}=H_{1}\left(F^{\prime}\right)$. Suppose that $\sigma_{n+1}(M)=h \in \mathrm{A}_{0}\left(F / F_{n+1}\right)$. Set $a_{i}=\rho\left(h\left(x_{i}\right)\right) \in F_{n}^{\prime}$, where $\rho: F \rightarrow F^{\prime}$ is the projection defined by $\rho\left(x_{i}\right)=1$. Then $\mu_{n}(S(M), p)=\sum_{i}\left[y_{i}\right] \otimes\left[a_{i}\right]$, where $\left[y_{i}\right] \in H^{\prime},\left[a_{i}\right] \in \mathrm{L}_{n}\left(H^{\prime}\right)$ are the classes represented by $y_{i}, a_{i}$. This assertion is just the obvious generalization of Corollary 2.2 and the proof is the same.

Now let $\sum_{i}\left[y_{i}\right] \otimes\left[\lambda_{i}\right]$ be an arbitrary element in $\mathrm{D}_{n}\left(H^{\prime}\right)$, where $\lambda_{i} \in F_{n}^{\prime}$, i.e. $\prod_{i}\left[y_{i}, \lambda_{i}\right] \in F_{n+2}$. We want to construct $(N, p)$ such that $\mu_{n}(N, p)=\sum_{i}\left[y_{i}\right] \otimes\left[\lambda_{i}\right]$. Consider the endomorphism $h$ of $F$ defined by

$$
\begin{aligned}
& h\left(x_{i}\right)=x_{i} \lambda_{i} \\
& h\left(y_{i}\right)=\lambda_{i}^{-1} y_{i} \lambda_{i} .
\end{aligned}
$$

Denote also by $h$ the induced automorphism of $F / F_{n+1}$. To see that $h \in \mathrm{A}_{0}\left(F / F_{n+1}\right)$, we compute

$$
\prod_{i}\left[h\left(x_{i}\right), h\left(y_{i}\right)\right]=\prod_{i}\left(x_{i} y_{i} x_{i}^{-1} \lambda_{i}^{-1} y_{i}^{-1} \lambda_{i}\right)=\prod_{i}\left[x_{i}, y_{i}\right]\left[y_{i}, \lambda_{i}^{-1}\right] \equiv \prod_{i}\left[x_{i}, y_{i}\right] \bmod F_{n+2} .
$$

Therefore, by Theorem $3, h=\alpha_{n}(M)$ for some $M \in \mathcal{H}_{g, 1}$. Since $\lambda_{i} \in F_{n}^{\prime}$ we have $h \in \mathrm{D}_{n}^{\mathrm{a}}(H)$ and so $M \in \mathcal{H}_{g, 1}[n]$. By the discussion above, $\mu_{n}(N, p)=\sum_{i}\left[y_{i}\right] \otimes\left[\lambda_{i}\right]$.

For completeness, we close this section by a sketch of a more direct proof of Theorem 4 using the results of $[\mathrm{O} 1, \mathrm{IO}]$. Similar arguments can be found in [CGO].

Lemma 4.8. For every $\alpha \in H_{3}\left(F / F_{n}\right)$ there is a closed 3-manifold $M$ and an n-equivalence $p: F \rightarrow \pi \stackrel{\text { def }}{=} \pi_{1}(M)$ such that $p_{*}[M]=\alpha$.

Remark 4.9. Since $\Omega_{3}\left(F / F_{n}\right) \cong H_{3}\left(F / F_{n}\right)$, it follows that every element $\alpha \in \Omega_{3}\left(F / F_{n}\right)$ is represented by some closed 3-manifold $M$ and map $p: \pi \stackrel{\text { def }}{=} \pi_{1}(M) \rightarrow F / F_{n}$ so that $p_{*}[M]=\alpha$. The point is to arrange that $p_{*}: H_{1}(M) \cong H_{1}\left(F / F_{n}\right)$, which would imply that $p$ is an $n$-equivalence.

Proof. We apply the constructions and results of [O1]. Consider the mapping cone $K_{n}$ of the natural map $K(F, 1) \rightarrow K\left(F / F_{n}, 1\right)$ of Eilenberg-MacLane spaces. $K_{n}$ is constructed from $K\left(F / F_{n}, 1\right)$ by adjoining 2-cells $e_{i}^{2}$ along the generators $x_{i} \in F \rightarrow F / F_{n}$. Then $K_{n}$ is simply-connected and $H_{i}\left(K_{n}\right) \cong H_{i}\left(F / F_{n}\right)$ for $i \geq 2$. So we have the Hurewicz epimorphism $\rho: \pi_{3}\left(K_{n}\right) \rightarrow H_{3}\left(K_{n}\right) \cong$ $H_{3}\left(F / F_{n}\right)$, where $\pi_{3}$ denotes the third homotopy group. Suppose $\rho(\theta)=\alpha$. Then the PontrjaginThom construction gives us a map $f: S^{3} \rightarrow K_{n}$ representing $\theta$, such that, if $x_{i} \in e_{i}^{2}$ is some interior 
point, then $f^{-1}\left(x_{i}\right)=L_{i}$, a zero-framed imbedded circle in $S^{3}$, see [O1]. Now let $M^{3}$ be the result of framed surgery on $S^{3}$ along the $\left\{L_{i}\right\}$. Then $f$ induces a map $p: M \rightarrow K\left(F / F_{n}\right)$ and it is clear that $p_{*}[M]=\alpha$. Finally we note that $p_{*}: H_{1}(M) \cong H_{1}\left(F / F_{n}\right)$.

From another viewpoint, we have defined a homomorphism $\mu_{n}^{\prime}: H_{3}\left(F / F_{n}\right) \rightarrow \mathrm{D}_{n}(H)$ by the formula

$$
\mu_{n}^{\prime}(\alpha)=\sum_{i, I} x_{i} \otimes\left(\alpha \frown\left(u_{i} \smile\left\langle u_{I}\right\rangle\right)\right) t_{I}
$$

and so, by Corollaries 2.2 and $4.3 \mu_{n}(M, p)=\mu_{n}^{\prime}\left(p_{*}[M]\right)$. It follows from Theorem 1 that the kernel of $\mu_{n}^{\prime}$ is precisely the image of $H_{3}\left(F / F_{n+1}\right) \rightarrow H_{3}\left(F / F_{n}\right)$, inducing, therefore, an injection $\operatorname{cok}\left(H_{3}\left(F / F_{n+1}\right) \rightarrow H_{3}\left(F / F_{n}\right)\right) \longmapsto \mathrm{D}_{n}(H)$. But it is shown in [O1, IO] that both sides are free finitely generated abelian groups of the same rank and so they are isomorphic. In fact K. Orr has pointed out to us that a straightforward examination of the spectral sequence of the group extension $F_{n} / F_{n+1} \rightarrow F / F_{n+1} \rightarrow F / F_{n}$ shows that the rank of $\operatorname{cok}\left(H_{3}\left(F / F_{n+1}\right) \rightarrow H_{3}\left(F / F_{n}\right)\right)$ is at least that of $\mathrm{D}_{n}(H)$.

4.3. Massey products and the Johnson homomorphism. In this section we will give a proof of Theorem 2. We first recall the definition of the Johnson homomorphism. Let $\mathrm{A}_{0}\left(F / F_{n}\right)$ be as in Section 2.4. We define the Johnson homomorphism

$$
\tau_{n}: \mathrm{A}_{0}\left(F / F_{n}\right) \rightarrow \operatorname{hom}\left(H, \mathrm{~L}_{n}(H)\right)
$$

where $H=H_{1}(F)$, as follows. If $\phi \in \mathrm{A}_{0}\left(F / F_{n}\right)$, then $\tau_{n}(\phi) \cdot[\alpha]=\alpha \phi\left(\alpha^{-1}\right) \in F_{n} / F_{n+1} \cong \mathrm{L}_{n}(H)$, where $[\alpha] \in H$ is the homology class of $\alpha \in F$. Let $K(\phi) \subseteq F$ be the normal closure of all elements of the form $\alpha \phi\left(\alpha^{-1}\right)$ for $\alpha \in F$; note that $K(\phi) \subseteq F_{n}$ if $\phi \in \mathrm{A}_{0}\left(F / F_{n}\right)$. Let $\pi=F / K(\phi)$. Then the projection $F \rightarrow \pi$ induces an isomorphism $p_{\phi}: F / F_{n} \cong \pi / \pi_{n}$ and the homomorphism $\hat{p}_{\phi}$ defined (as $\hat{p}$ ) in Corollary 4.3 is given by the composition

$$
H_{2}(\pi) \cong K(\phi) /[F, K(\phi)] \rightarrow F_{n} / F_{n+1} \cong \mathrm{L}_{n}(H)
$$

The first isomorphism is given by Hopf's theorem. Now consider the natural homomorphism $i: H \rightarrow H_{2}(\pi)$ defined by $i([\alpha])=\left[\alpha \phi(\alpha)^{-1}\right]$, then

$$
\tau_{n}(\phi)=\hat{p}_{\phi} \circ i \text {. }
$$

Now let $\Sigma$ be a compact orientable surface of genus $g$ with one boundary component. Then $\pi_{1}(\Sigma) \cong F$, the free group on $2 g$ generators. By Nielsen's theorem $\Gamma_{g, 1} \subseteq \mathrm{A}(F)$ and so $\Gamma_{g, 1}[n]=$ $\Gamma_{g, 1} \cap \mathrm{A}_{0}\left(F / F_{n}\right)$.

For $\phi \in \Gamma_{g}$ define an associated closed 3-manifold $T_{\phi}$ as follows. Let $T_{\phi}^{\prime}$ be the mapping torus of $\phi$, i.e. $I \times \Sigma$ with $1 \times \Sigma$ identified with $0 \times \Sigma$ by the homeomorphism $(1, x) \rightarrow(0, \phi(x))$ for every $x \in \Sigma$. Since $\phi \mid \partial \Sigma=$ id there is a canonical identification of $\partial T_{\phi}^{\prime}$ with the torus $S^{1} \times S^{1}$. $T_{\phi}$ is defined by pasting in $D^{2} \times S^{1}$. Note that $\pi_{1}\left(T_{\phi}\right) \cong F / K(\phi)$, where $K(\phi)$ was defined above.

A theorem of the following sort was first suggested by Johnson [Jo1] and a proof (which we sketch, for completeness) was given in [Ki].

Proposition 4.10. $\tau_{n}(\phi)=\mu_{n}\left(T_{\phi}\right)$, where $H_{1}(\Sigma)$ and $H^{1}\left(T_{\phi}\right)$ are identified by the string of isomorphisms

$$
H^{1}\left(T_{\phi}\right) \stackrel{\cong}{\rightrightarrows} H^{1}(\Sigma) \cong H_{1}(\Sigma)
$$

induced by the inclusion $\Sigma \subseteq T_{\phi}$ and Poincaré duality for $\Sigma$.

Proof. It follows from the definitions that we need to establish the commutativity of the following diagram 


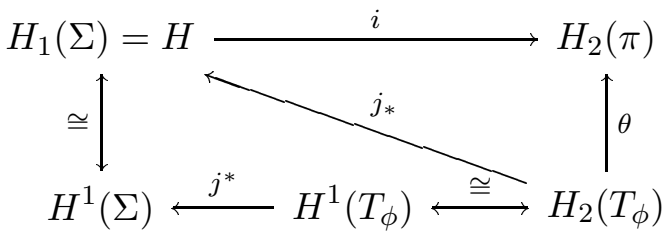

where $i$ is defined in equation (14), $j: \Sigma \subseteq I \times 0 \subseteq I \times \Sigma \rightarrow T_{\phi}$ is the inclusion map inducing $j^{*}$ and the Gysin homomorphism $j_{*}$, and $\theta$ is the Hopf map $H_{2}(X) \rightarrow H_{2}\left(\pi_{1}(X)\right)$ when $X=T_{\phi}$.

Now suppose $\alpha \in \pi_{1}(\Sigma)$ is represented by a 1-cycle $z$ in $\Sigma$. since $\phi(z)$ is homologous to $z$, there exists a 2-chain $c$ in $\Sigma$ such that $\partial c=\phi(z)-z$. Consider the chain $I \times z$ in $I \times \Sigma \rightarrow T_{\phi}$ with boundary $1 \times z-0 \times z$. Since $0 \times z$ is identified with $1 \times \phi(z)$ in $T_{\phi}$, the chain $\xi=I \times z+1 \times c$ is a 2-cycle in $T_{\phi}$ - let $\beta$ denote its homology class in $H_{2}\left(T_{\phi}\right)$.

It follows from the definition of $\theta$ that $\theta(\beta)=i([\alpha])$. On the other hand $j_{*}(\beta)=[\alpha]$ since the 2-cycle $\xi$ intersects $\epsilon \times \Sigma$ transversely in the 1-cycle $\epsilon \times z$, if $0<\epsilon<1$. This establishes the desired commutativity.

Combining Proposition 4.10 and Corollary 4.3 we have

Corollary 4.11. If $\left\{x_{i}\right\}$ is a basis of $H=H_{1}(\Sigma) \cong H^{1}\left(T_{\phi}\right)$ and $\left\{u_{i}\right\}$ is the dual basis of $H_{1}\left(T_{\phi}\right)$, then

$$
\tau_{n}(h)=\sum_{i, I} x_{i} \otimes\left(\left[T_{\phi}\right] \frown\left(u_{i} \smile\left\langle u_{I}\right\rangle\right)\right) t_{I}
$$

using the inclusion $\mathrm{L}_{n}(H) \subseteq \mathbb{Z} \llbracket t_{1}, \ldots, t_{2 g} \rrbracket_{n}$.

We now turn to the proof of Theorem 2. Fix an $n$-equivalence $p: F \rightarrow \pi_{1}(M)$, an imbedding $\iota: \Sigma \rightarrow M$ of a (closed) surface in a (closed) 3-manifold $M$, and an element $\phi \in \Gamma_{g}[n]$. The homomorphisms $f: \pi_{1}(M) \rightarrow F / F_{n+1}$ and $f_{\phi}: \pi_{1}\left(M_{\phi}\right) \rightarrow F / F_{n+1}$ induced by the $n$-equivalence $p$ (in the discussion before the statement of the theorem) determine maps $f: M \rightarrow K\left(F / F_{n+1}, 1\right)$ and $f_{\phi}: M_{\phi} \rightarrow K\left(F / F_{n+1}, 1\right)$ in the Eilenberg-MacLane space $K\left(F / F_{n}, 1\right)$. We will construct a cobordism $F: V^{4} \rightarrow K\left(F / F_{n+1}\right)$ between $f$ and $f_{\phi}$.

First we push $\iota(\Sigma)$ in the positive and negative normal directions in $M$ to obtain two copies of $\iota(\Sigma), \Sigma_{p}$ and $\Sigma_{n}$, respectively. Then we attach $I \times \iota(\Sigma)$ to $M$ along its boundary by identifying $0 \times \iota(\Sigma)$ to $\Sigma_{n}$ by the homeomorphism $(0, x) \rightarrow x$, for $x \in \iota(\Sigma)$, and $1 \times \iota(\Sigma)$ to $\Sigma_{p}$ by $(1, x) \rightarrow \phi(x)$. We can now thicken up $I \times \iota(\Sigma)$ and $M$ to obtain a manifold $W$, see Figure 2 .

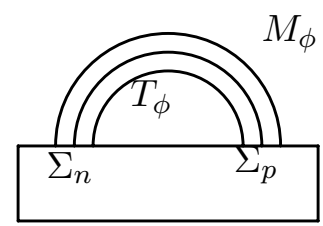

$M$

Figure 2. The manifold $W$. The handle shown represents a thickening of $\iota(\Sigma)$, shown as the core.

Note that the boundary of $W$ consists of three components: $M, M_{\phi}$ and the mapping torus $T_{\phi}$ of $\phi$ on $\iota(\Sigma)$. We make one small modification to obtain $V$. Remove a disk $D$ from $\iota(\Sigma)$ to obtain a surface $\Sigma^{o}$ with boundary and lift $\phi$ to a diffeomorphism $\phi^{o}$ of $\Sigma^{o}$. Then $S^{1} \times D$ is naturally imbedded in $T_{\phi}$, and so in the boundary of $W$. We attach $D^{2} \times D$ to $W$ along $S^{1} \times D$ to obtain $V$.

The boundary of $V$ is now given by $\partial V=M_{\phi}-T_{\phi^{o}}-M$. It is not difficult to check that the inclusions $M \rightarrow V \leftarrow M_{\phi}$ are $(n+1)$-equivalences since since $\phi \in \Gamma_{g}[n+1]$. Then $f, f_{\phi}$ extend to 
a map $F: V \rightarrow K\left(F / F_{n+1}, 1\right)$. In addition the inclusions $\Sigma^{o} \subseteq T_{\phi^{o}} \subseteq V$ induces isomorphisms

$$
F / F_{n+1} \cong \pi_{1}\left(\Sigma^{o}\right) / \pi_{1}\left(\Sigma^{o}\right)_{n+1} \cong \pi_{1}\left(T_{\phi^{o}}\right) / \pi_{1}\left(T_{\phi^{o}}\right)_{n+1} \cong \pi_{1}(V) / \pi_{1}(V)_{n+1}
$$

So the bordism $(F, V)$ implies that $\left(f_{\phi}\right)_{*}\left[M_{\phi}\right]=f_{*}[M]+\left(p_{\phi}\right)_{*}\left[T_{\phi^{o}}\right] \in \Omega_{3}\left(F / F_{n+1}\right) \cong H_{3}\left(F / F_{n+1}\right)$.

Applying the map of Equation (1) concludes the proof of Theorem 2.

Remark 4.12. Theorem 2 and Proposition 4.10 can be generalized easily to the context of homology cylinders, by essentially the same arguments. For any homology cylinder $N$ there is an obvious notion of mapping torus $T_{N}$ - then Proposition 4.10 can be rephrased, replacing $T_{\phi}$ by $T_{N}, \phi \in$ $\Gamma_{g, 1}[n]$ by $N \in \mathcal{H}_{g, 1}[n]$ and $\tau_{n}$ by $\varsigma_{n}$. For Theorem 2 we consider $N \in \mathcal{H}_{g, 1}$ and an imbedding $\Sigma \subseteq M$, where $\Sigma$ is a closed orientable surface of genus $g$. We can cut $M$ open along $\Sigma$ and paste in $\bar{N}$-where $\bar{N}$ is obtained from $N$ by filling it in, in the obvious way, to get a homology cylinder over $\Sigma$-and so obtain a new manifold $M_{N}$. Then Theorem 2 can be rephrased, replacing $M_{\phi}$ by $M_{N}$.

Remark 4.13. We can consider another filtration of $\mathcal{H}_{g, 1}$. Let $\mathcal{H}_{g, 1}(n)$ denote the set of all homology cylinders $n$-equivalent to $\Sigma_{g, 1} \times I$ (see Section 2.5). Thus if $M \in \mathcal{H}_{g, 1}(n)$ then $M-\left(\Sigma_{g, 1} \times I\right) \in$ $\mathcal{F}_{n}^{Y}\left(\Sigma_{g, 1} \times I\right)$. It is easy to see that $\mathcal{H}_{g, 1}(n)$ is a subsemigroup of $\mathcal{H}_{g, 1}$. It is a natural conjecture that the quotient $\mathcal{H}_{g, 1} / \mathcal{H}_{g, 1}(n)$ is a group.

According to [GGP] we get the same filtration if we ask that $M$ be obtained from $\Sigma_{g, 1} \times I$ by cutting open along some imbedded closed orientable surface $\Sigma^{\prime} \subseteq \Sigma_{g, 1} \times I$ and reattaching by some element of $\mathcal{T}_{n}$, the $n$-th lower central series subgroup of the Torelli group $\mathcal{T}$ of $\Sigma^{\prime}$. It is clear that $\mathcal{H}_{g, 1}(n) \subseteq \mathcal{H}_{g, 1}[n]$ since the effect of cutting and reattaching in $M$ by an element of $\mathcal{T}_{n}$ does not change $\pi_{1}(M) / \pi_{1}(M)_{n}$. If $\mathcal{G H}_{g, 1}(*)$ and $\mathcal{G H}_{g, 1}[*]$ denote the associated graded groups of these filtrations then we have a natural map $\mathcal{G H}_{g, 1}(*) \rightarrow \mathcal{G} \mathcal{H}_{g, 1}[*]$. Note also that the natural homomorphism $\Gamma_{g, 1} \rightarrow \mathcal{H}_{g, 1}$ induces maps $\left(\mathcal{T}_{g, 1}\right)_{n} \rightarrow \mathcal{H}_{g, 1}(n)$ and so $\mathcal{G}\left(\mathcal{T}_{g, 1}\right)_{*} \rightarrow \mathcal{G H}_{g, 1}(*)$.

Putting this, and some of the other maps constructed in this paper, all together, we have a commutative diagram:

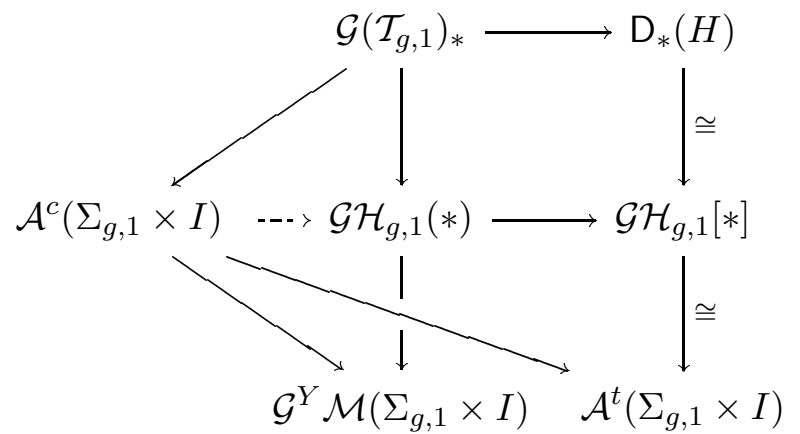

The dotted arrow denotes a conjectured lifting.

\section{Questions}

It is well known that there is a set of moves that generates (string) link concordance, [Tr]. These moves, together with the existence of the Kontsevich integral, were the key to the proof that the tree-level part of the Kontsevich integral of string-links is given by Milnor's invariants, or equivalently, by Massey products, see [HM].

Question 1. Is there a set of local moves that generates homology cobordism of homology cylinders?

Question 2. Does Theorem 6 generalize to homology cylinders, using our extension of the Johnson homomorphism? See Remark 4.13. 
A positive answer to the above question would imply that the full tree-level part of the theory of finite type invariants on 3-manifolds is given by our extension of the Johnson homomorphism to homology cylinders.

Question 3. If $\left(\Sigma_{g, 1} \times I\right)-M \in \mathcal{F}_{n}^{Y}\left(\Sigma_{g, 1} \times I\right)$, is $M \in \mathcal{H}_{g, 1}(n) \otimes \mathbb{Q}$ ? See Remark 4.13. When $g=0$ the answer is yes - see [GL2]. Are the $\mu$-invariants of homology cylinders (see Remark 2.4) finite-type in the Goussarov-Habiro sense?

We now consider the group $\mathcal{H}_{g, 1}^{c}$ of homology cobordism classes of homology cylinders defined in Remark 2.3. The subgroup $\mathcal{H}_{g, 1}^{c}[2]$ (see Proposition 2.5) is the analogue of the Torelli group, which we denote $\mathcal{T} \mathcal{H}_{g, 1}^{c}$. We can consider the lower central series filtration $\left(\mathcal{T} \mathcal{H}_{g, 1}^{c}\right)_{n}$ and, just as in the case of the Torelli group (see [Mo3]), we have $\left(\mathcal{T H}_{g, 1}^{c}\right)_{n} \subseteq \mathcal{H}_{g, 1}^{c}[n+1]$. Recall also that Hain $[\mathrm{Ha}]$ proved that, over $\mathbb{Q}$, the associated graded Lie algebra of the lower central series filtration of the Torelli group maps onto the associated graded Lie algebra of the weight filtration of the Torelli group, and that these two filtrations are known to differ in degree 2 by a factor of $\mathbb{Q}$, [Ha, Mo4]. Whether they differ in degrees other than 2 is an interesting question.

Question 4. What is the relation between the filtrations $\left(\mathcal{T H}_{g, 1}^{c}\right)_{n}$ and $H_{g, 1}^{c}[n]$ ?

Notice that the answer to above question is not known for the group of concordance classes of string-links, see [HM], but it is known (in the positive) for the group of homotopy classes of string-links, see [HL], and for the pure braid group, see [Kh].

Question 5. We have $\left(\mathcal{T}_{g, 1}\right)_{n} \subseteq \mathcal{H}_{g, 1}^{c}(n)$ (see Remark 4.13) and obviously $\left(\mathcal{T}_{g, 1}\right)_{n} \subseteq\left(\mathcal{T H}_{g, 1}^{c}\right)_{n}$. What is the relation between the filtrations $\mathcal{H}_{g, 1}^{c}(n)$ and $\left(\mathcal{T H}_{g, 1}^{c}\right)_{n}$ ?

We now consider the center $\mathcal{Z}\left(\mathcal{H}_{g, 1}^{c}\right)$ of $\mathcal{H}_{g, 1}^{c}$. This contains, at least, the group $\theta^{H}$ of homology cobordism classes of homology 3-spheres (see Remark 2.3). Furthermore $\mathcal{Z}\left(\mathcal{H}_{g, 1}^{c}\right)$ contains also the element $\tau_{\partial}$ defined by a Dehn twist about the boundary of $\Sigma_{g, 1}$. For the mapping class group $\Gamma_{g}$ it seems to be true (according to J. Birman and C. McMullen) that the center is trivial, at least for large $g$.

Question 6. Determine $\mathcal{Z}\left(\mathcal{H}_{g, 1}^{c}\right)$. Is it generated by $\theta^{H}$ and $\tau_{\partial}$ ?

We now consider the subgroup $\mathcal{H}_{g, 1}^{c}[\infty] \subseteq \mathcal{H}_{g, 1}^{c}$. This contains $\theta^{H} \times \mathcal{S}_{g}^{c}[\infty]$, where $\mathcal{S}_{g}^{c}[\infty]$ denotes the subgroup of the string-link concordance group $\mathcal{S}_{g}^{c}$ consisting of string links with vanishing $\mu$ invariants (see Remarks 2.3 and 2.7). $\mathcal{S}_{g}^{c}[\infty]$ contains, for example, all boundary string links and, in particular, the knot concordance group, which is an abelian group of infinite rank (see[Le1]).

Question 7. Determine $\mathcal{H}_{g, 1}^{c}[\infty]$. Is it equal to $\theta^{H} \times \mathcal{S}_{g}^{c}[\infty]$ ?

Question 8. Is $\mathcal{Z}\left(\mathcal{H}_{g, 1}^{c}\right) / \mathcal{Z}\left(\mathcal{H}_{g, 1}^{c}\right) \cap \mathcal{H}_{g, 1}^{c}[\infty]$ the infinite cyclic group generated by $\tau_{\partial}$ ?

In [Mo4], Morita calculated the symplectic invariant part $\mathrm{D}(H)^{\mathfrak{s p}}$ of $\mathrm{D}(H)$ in terms of a beautiful space of chord diagrams. The group $\mathcal{Z}\left(\mathcal{H}_{g, 1}^{c}\right) /\left(\mathcal{Z}\left(\mathcal{H}_{g, 1}^{c}\right) \cap \mathcal{H}_{g, 1}^{c}[\infty]\right)$ is closely related to $\mathrm{D}(H)^{\mathfrak{s p}}$, since the image under the map $\sigma_{n}$ of Theorem 3 of an element in $\mathcal{Z}\left(\mathcal{H}_{g, 1}^{c}\right) \cap \mathcal{H}_{g, 1}^{c}[n]$ lies in $\mathrm{D}_{n}(H)^{\mathfrak{s p}}$. Thus any element of $\mathcal{Z}\left(\mathcal{H}_{g, 1}^{c}\right) / \mathcal{Z}\left(\mathcal{H}_{g, 1}^{c}\right) \cap \mathcal{H}_{g, 1}^{c}[\infty]$ provides a geometric construction of an element of $\mathrm{D}(H)^{\mathfrak{s p}}$.

The following question is important to the philosophical notion of finite type.

Question 9. Is $\mathcal{H}_{g, 1}^{c}$ finitely-generated? Is its abelianization finitely-generated? Note that both the mapping class group $\Gamma_{g, 1}$ and the Torelli group $\mathcal{T}_{g, 1}$ are finitely-generated. Note also that $\mathcal{S}_{g}^{c}$ and $\theta^{H}$ are infinitely-generated abelian (see $[\mathrm{F}]$ ) and, as for the analogous question for string-links, since the knot concordance group has infinite rank, the abelianization of the string-link concordance group (on any number of strings) has infinite rank.

Question 10. Let $\mathcal{H}_{g}$ denote the semigroup of homology cylinders over the closed surface $\Sigma_{g}$ of genus $g$. The kernel of the obvious epimorphism $\mathcal{H}_{g, 1} \rightarrow \mathcal{H}_{g}$ is related to concordance classes 
of framed proper arcs in $I \times \Sigma_{g}$. Describe this more explicitly and consider also the homology cobordism groups $\mathcal{H}_{g}^{c}$.

5.1. Note. The present paper was completed in 1999, and its follow-up by the second author appeared in [Le3].

\section{REFERENCES}

[AMR1] J. Andersen and J. Mattes and N. Reshetikhin, The Poisson structure on the moduli space of flat connections and chord diagrams, Topology 35 (1996) 1069-1083.

[AMR2] , Quantization of the algebra of chord diagrams, Math. Proc. Cambridge Phil. Soc. 124 (1998) 451-467.

[B-N] D. Bar-Natan, Vassiliev homotopy string-link invariants, J. Knot Theory and its Ramifications 4 (1995) 13-32.

[CE] H. Cartan, S. Eilenberg, Homological Algebra, Princeton University Press, 1956.

[CGO] T. Cochran, A. Gerges and K. Orr, Dehn surgery equivalence relations on three-manifolds, Math. Proc. Cambridge Philos. Soc. 131 (2001) 97-127.

[Dr] V. G. Drinfeld, On quasitriangular quasi Hopf algebras and a group closely connected with Gal $(\overline{\mathbb{Q}} / \mathbb{Q})$, Leningrad Math. J. 2 (1991) 829-860.

[Dw] W. Dwyer, Homology, Massey products and maps between groups, J. Pure and Applied Algebra 6 (1975) 177-190.

[FS] R. Fenn, D. Sverje, Massey products and lower central series of groups, Canad. J. Math. 39 (1987) 322-337.

[F] M. Furuta, Homology cobordism group of homology 3-spheres, Inventiones Math. 100 (1990) 339-355.

[GGP] S. Garoufalidis, M. Goussarov and M. Polyak, Calculus of clovers and finite type invariants of 3-manifolds, Geometry and Topology, 5 (2001) 75-108.

[GL1] S. Garoufalidis and J. Levine, Finite type 3-manifold invariants, the mapping class group and blinks, J. Diff. Geom. 47 (1997) 257-320.

[GL2] - Finite type 3-manifold invariants and the Torelli group I, Inventiones Math. 131 (1998) 541-594.

[Gu1] M. Goussarov, Finite type invariants and n-equivalence of 3-manifolds, C. R. Acad. Sci. Paris Ser. I. Math. 329 (1999) 517-522.

[Gu2] Wariations of knotted graphs, geometric technique of n-equivalence, St. Petersburg Math. J. 12 (2001).

[HL] N. Habegger and X-S. Lin, The classification of links up to homotopy, Journal AMS 3 (1990) 389-419.

[HM] _ and G. Masbaum, The Kontsevich Integral and Milnor's Invariants, Topology 39 (2000) $1253-1289$.

[Hb] K. Habiro, Clasper theory and finite type invariants of links, Geometry and Topology 4 (2000), 1-83.

[Ha] R. Hain, Infinitesimal presentations of the Torelli groups, Journal of AMS 10 (1997) 597-651.

[Ho] H. Hopf, Fundamentalgruppe und Zweite Bettische Gruppe, Comment. Math. Helvetici 14 (1942) $257-309$.

[IO] K. Igusa, K. Orr, Links, pictures and the homology of nilpotent groups, Topology 40 (2001) 1125-1166.

[Ih] Y. Ihara, Some problems on three-point ramifications and associated large Galois representations, Adv. Studies in Pure Math. 12 North-Holland (1987) 173-188.

[Jo1] D. Johnson, An abelian quotient of the mapping class group, Math. Ann. 249 (1980) 225-242.

[Jo2] _ A survey of the Torelli group, Contemporary Math. 20 (1983) 163-179.

[KM] M. Kervaire, J.W. Milnor, Groups of homotopy spheres I, Annals of Math. 77 (1963) 504-537.

[Ki] T. Kitano, Johnson's homomorphisms of subgroups of the mapping class group, the Magnus expansion and Massey higher products of mapping tori, Topology and its Applic. 69 (1996) 165-172.

[Kh] T. Kohno, Série de Poincaré-Koszul associée aux groupes de Tresses Pures, Inventiones Math. 82 (1985) $57-76$.

[Ko1] M. Kontsevich, Formal (non)-commutative symplectic geometry, Gelfand Math. Seminars, 1990-92, Birkhauser, Boston, (1993) 173-188.

[Ko2] _ Feynmann diagrams and low-dimensional topology, Proceedings of the first European Congress of Mathematicians, vol. 2, Progress in Math. 120 Birkhauser, Boston, (1994) 97-121.

[Ko3] _ Operads and motives in deformation quantization, Lett. Math. Phys. 48 (1999) 35-72.

[Kr] D. Kraines, Massey higher products, Transactions AMS 124 (1966) 431-449.

[LMO] T.T.Q. Le, J. Murakami, T. Ohtsuki, A universal quantum invariant of 3-manifolds, Topology 37 (1998) $539-574$.

[La] L. Lambe, Two exact sequences in rational homotopy theory relating cup products and commutators, Proc. AMS 96 (1986) 360-364.

[Le1] J. Levine, Invariants of knot cobordism, Inventiones Math. 8 (1969) 98-110. 
[Le2] L Link concordance and algebraic closure, Comment. Math. Helvetici 64 (1989) 236-255.

[Le3] Homology cylinders: an enlargement of the mapping class group, Algebr. Geom. Topol. 1 (2001) 243-270.

[Mac] S. MacLane, Homology, Springer-Verlag, 1963.

[MKS] W. Magnus, A. Karras and D. Solitar, Combinatorial group theory, presentations of groups in terms of generators and relations, Wiley, New York, 1966.

[Ma] W.S. Massey, Higher order linking numbers, J. Knot Theory and its Ramifications 7 (1998) 393-414.

[M] S. V. Matveev, Generalized surgery of three-dimensional manifolds and representations of homology spheres, Math. Notices Acad. Sci. USSR, 42:2 (1987) 651-656.

[MM] J.W. Milnor and J.C. Moore, On the structure of Hopf algebras, Ann. Math. 81 (1965) 211-264.

[Mo1] S. Morita, Casson's invariant for homology 3-spheres and characteristic classes of vector bundles I, Topology 28 (1989) 305-323.

[Mo2] Abelian quotients of subgroups of the mapping class group of surfaces, Duke Math. Journal 70 (1993) 699-726.

[Mo3] A linear representation of the mapping class group of orientable surfaces and characteristic classes of vector bundles, Topology of Teichmüller spaces, S. Kojima et al editors, World Scientific, (1996) 159-186.

[Mo4] Structure of the mapping class group of surfaces: a survey and a prospect, Proceedings of the Kirbyfest Geom. Topol.Monogr., 2 (1999) 349-406.

[Oh] T. Ohtsuki, A filtration of the set of integral homology 3-spheres, Proceedings of the International Congress of Mathematicians, Vol. II, Berlin (1998) 473-482.

[O1] K. Orr, Homotopy invariants of links, Inventiones Math. 95 (1989) 379-394.

[O2] L Link concordance invariants and Massey products, Topology 30 No. 4 (1991) 699-710.

[Qu] D. Quillen, On the associated graded ring of a group ring, Journal of Algebra 10 (1968) 411-418.

[St] J. Stallings, Homology and central series of groups, Journal of Algebra 2 (1965) 170-181.

[Su] D. Sullivan, On the intersection ring of compact 3-manifolds, Topology 14 (1975) 275-277.

[Tr] A. Tristram, Some cobordism invariants for links, Math. Proc. Cambridge Phil. Soc. 66 (1969) $251-264$.

[Tu] V. G. Turaev, Nilpotent homotopy types of closed 3-manifolds, Proceedings of Topology Conference in Leningrad, Lecture Notes in Mathematics 1060 (1982), Springer-Verlag, 355-366.

School of Mathematics, Georgia Institute of Technology, Atlanta, GA 30332-0160, USA, http://www.math.gatech .edu/ stavros

E-mail address: stavros@math.gatech.edu

Department of Mathematics, Brandeis University, Waltham, MA 02254-9110, USA.

E-mail address: levine@brandeis.edu 